\begin{tabular}{|l|l|}
\hline $\begin{array}{l}\text { 2. To: (Receiving Organization) } \\
\text { Distribution }\end{array}$ & $\begin{array}{l}\text { 3. From: (Originating Organization) } \\
\text { Retrieval Engineering }\end{array}$ \\
\hline $\begin{array}{l}\text { 5. Proj/Prog/Dept./Div.: } \\
\text { RPP/WFD }\end{array}$ & $\begin{array}{l}\text { 6. Dedign Authority/Design Agent/Cog. Engr.: } \\
\text { J. R. Jewett, 373-1228 }\end{array}$ \\
\hline
\end{tabular}

\section{Originator Remark:}

Document transmitted for approval and release.

\section{Related EDT No.: \\ NA \\ 7. Punchase Order No.: \\ NA}

9. Equip./Compononk No.:

NA

10. Syatem/Bidg./Faciliky:

Tank Farms

12. Major Aaen. Dwg. No.:

NA

13. Permit/Permit Application No::

NA

14. Required Response Date:

NA

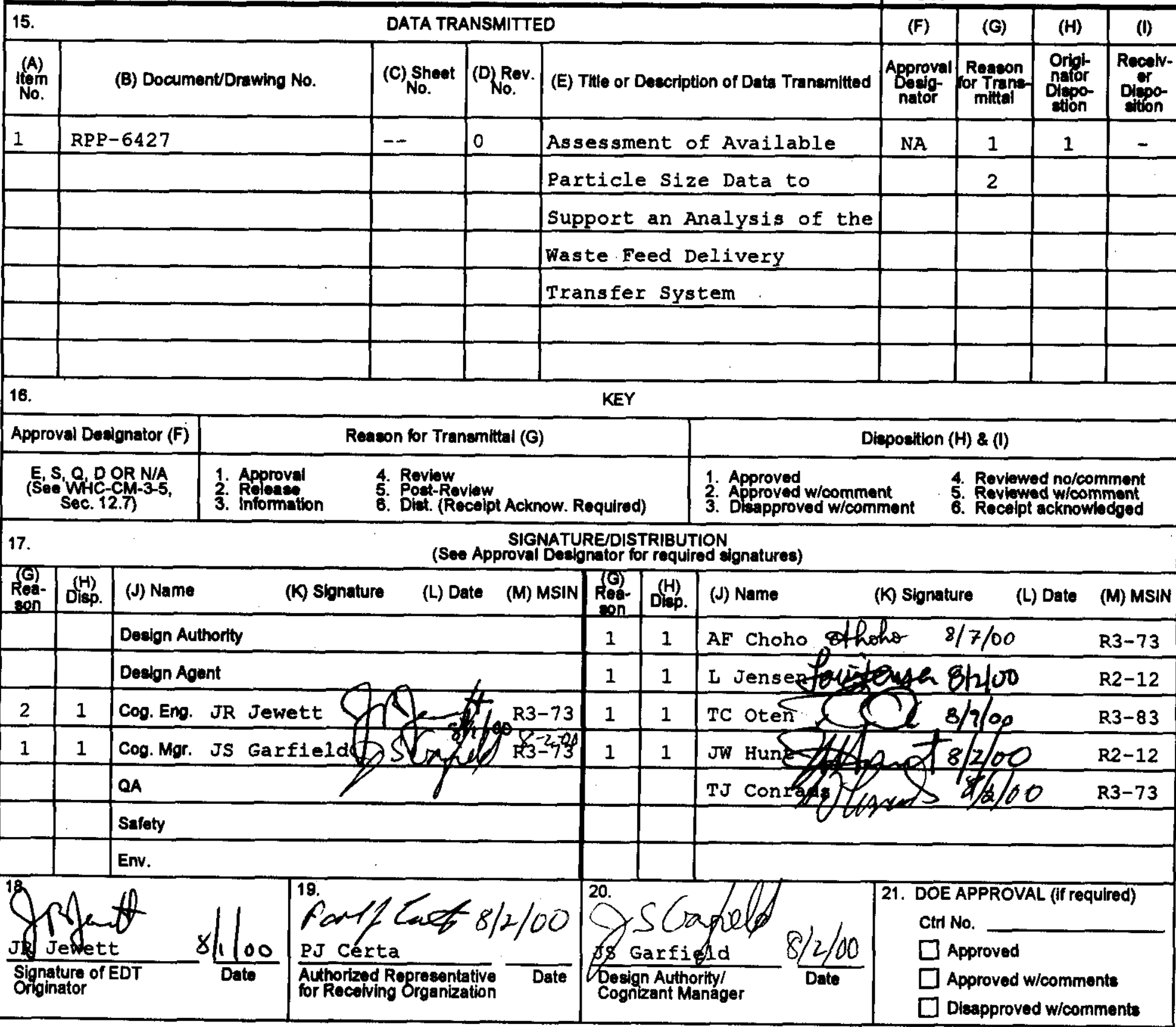




\section{DISTRIBUTION}

\section{Onsite}

11

CH2M HILL Hanford Group, Inc.

Barton, W. B., III

R2-11

Bechtold, R. A.

K7-22

Field, J. G.

R2-12

Hunt, J. W.

R2-12

Jensen, L. (3)

R2-12

Julyk, L. J.

R3-83

Kirch, N. W.

R2-11

Oten, T. C.

R3-83

Parker, D. L.

R3-75

5

Lockheed Martin Services, Inc.

Rice, R. L. (3)

R3-73

Central Files

B1-07

Document Processing Center

Numatec Hanford Corporation

Carlson, A. B.

R3-73

Certa, P. J.

R3-73

Choho, A. F.

R3-73

Conrads, T. J.

R3-73

Galbraith, J. D.

R3-73

Garfield, J. S.

R3-73

Grenard, C. E.

R3-73

Jewett, J. R. (5)

R3-73

Senentz, G.

R3-73

Willis, W. L.

R3-73

Subcontractors

Bechtold, D. B., ANL SVC

T6-07

Callaway, W. S., III, COGEMA

Herting, D. L., ANL SVC

T6-07

Lockrem, L. L., ANL SVC

T6-07

U.S. Department of Energy Richland Operations Office

Reading Room

$\mathrm{H} 2-53$

Distr.-1 


\section{DISTRIBUTION (continued)}

Offsite

1

Oak Ridge National Laboratory

C. P. McGinnis

Oak Ridge National Laboratory

P.O. Box 2008, MS6178

Oak Ridge, Tennessee 37831-6178

1

Washington State University

K. C. Liddell

Department of Chemical Engineering

Washington State University

Pullman, Washington 99164-2710

Distr.-2 


\title{
Assessment of Available Particle Size Data to Support an Analysis of the Waste Feed Delivery System Transfer System
}

\author{
J. R. Jowett, Numatec Banford Corporation \\ L. Jensen, CH2M HILL Hanford Group, Inc. \\ Richland, WA 99352 \\ U.S. Department of Energy Contract DE-AC06-99RL14047

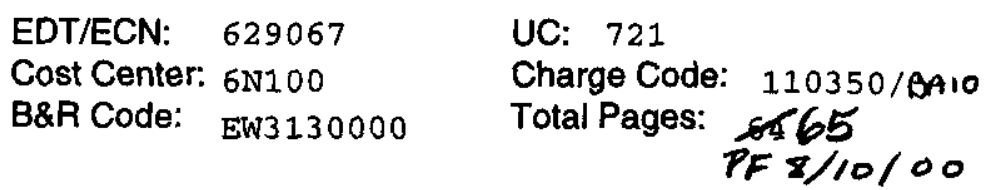

Key Words: particle size distribution analysis, slurry transport, waste feed delivery, high-level waste, low-activity waste, tank waste, particle size measurement

Abstract: Available data pertaining to size distribution of the particulates in Hanford underground tank waste have been reviewed. Although considerable differences exist between measurement methods, it may be stated with $95 \%$ confidence that the median particle size does not exceed $275 \mu \mathrm{m}$ in at least 958 of the ten tanks selected as sources of HLW feed for Phase 1 vitrification in the RPP. This particle size is recommended as a design basis for the WFD transfer system.

TRADEMARK DISCLAIMER. Reference herein to any specific commercial product, process, or service by trade name, trademark, manufacturer, or otherwise, does not necessarily constitute or imply its endorsement, recommendation, or favoring by the United States Government or any agency thereof or its contractors or subcontractors.

Printed in the United States of America. To obtain copies of this document, contact: Document Control Services, P.O. Box 950, Mailstop H6-08, Richland WA 99352, Phone (509) 372-2420; Fax (509) 376-4989.
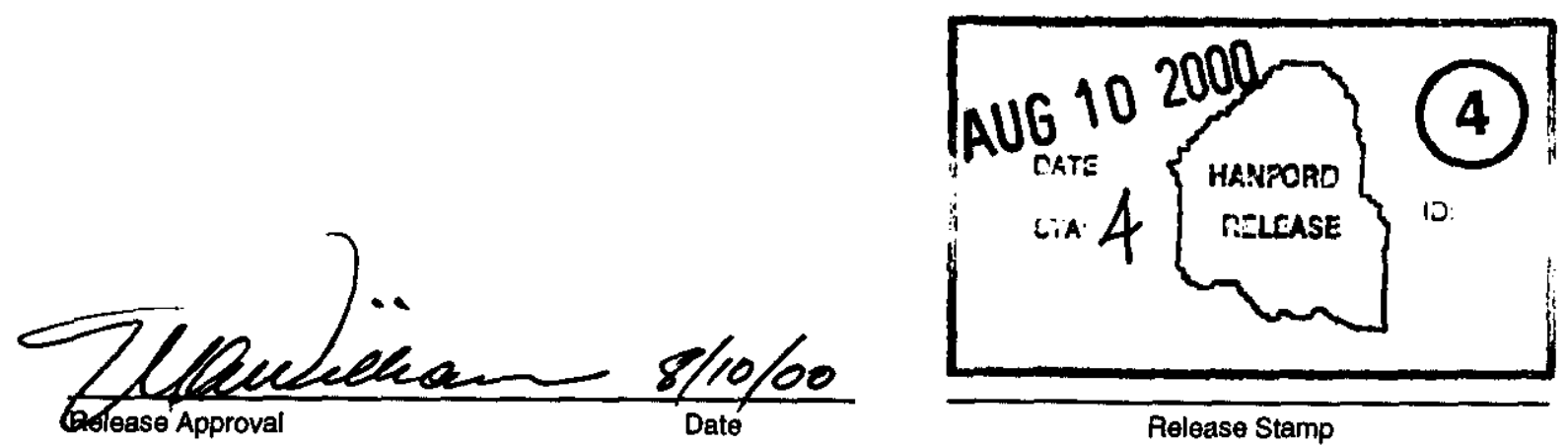

\section{Approved For Public Release}




\section{Assessment of Available Particle Size Data to Support an Analysis of the Waste Feed Delivery System Transfer System}

Prepared for the U.S. Department of Energy

Assistant Secretary for Environmental Management

\section{CH2MHILL \\ Hanford Group, Inc.}

Richland, Washington

Contractor for the U.S. Department of Energy

Office of River Protection under Contract DE-AC06-99RL14047

Approved for Public Release; Further Dissemination Unlimited 


\section{Assessment of Available Particle Size Data to Support an Analysis of the Waste Feed Delivery System Transfer System}

Prepared by:

J. R. Jewett

Numatec Hanford Corporation

L. Jensen

CH2M HILL Hanford Group, Inc.

Date Published:

August 2000

\section{CH2MHILL}

Hanford Group, Inc.

P. O. Box 1500

Richland, Washington

Contractor for the U.S. Department of Energy

Office of River Protection under Contract DE-ACO6-99RL14047 
LEGAL DISCLAIMER

This report was prepared as an account of work sponsored by an agency of the United States Government. Neither the United States Government nor any agency thereof, nor any of their employees, nor any of their contractors, subcontractors or their employees, makes any warranty, express or implied, or assumes any legal liability or responsibility for the accuracy, completeness, or any third party's use or the results of such use of any information, apparatus, product, or process disclosed, or represents that its use would not infringe privately owned rights. Reference herein to any specific commercial product, process, or service by trade name, trademark, manufacturer, or otherwise, does not necessarily constitute or imply its endorsement, recommendation, or favoring by the United States Government or any agency thereof or its contractors or subcontractors. The views and opinions of authors expressed herein do not necessarily state or reflect those of the United States Government or any agency thereof.

This report has been reproduced from the best available copy.

Available in paper copy and microfiche.

Available electronically at http://www.doe.gov/bridge. Available for a processing fee to the U.S. Department of Energy and its contractors, in paper, from:

U.S. Department of Energy

Office of Scientific and Technical Information

P.O. Box 62

Oak Ridge, TN 37831-0062

phone: 865-576-8401

fax: 865-576-5728

email: reports@adonis,osti.gov(423) 576-8401

Available for sale to the public, in paper, from:

U.S. Department of Commerce

National Technical Information Service

5285 Port Royal Road

Springfield, VA 22161

Phone: 800-553-6847

fax: 703-605-6900

omail: orders@ ntis.fedworld.gov

online ordering:

http://www.ntis.gov/ordering.htm 


\section{RPP-6427 REV 0}

This page intentionally left blank. 


\section{RPP-6427 REV 0}

\section{EXECUTIVE SUMMARY}

As part of the Waste Feed Delivery Program, an engineering analysis (RPP-5346) was performed to determine the adequacy of the waste transfer system (piping, pumps, valves, and fittings) at the Hanford Site. For each transfer anticipated during Phase 1 of the River Protection Project, the velocity of the waste required to suspend and transport the solid fraction of the waste was determined. The pipeline pressure required to achieve this velocity was determined also. The analysis was hampered by a lack of certainty about the size of the particles present in the high-level waste (HLW) slurries to be delivered by the Waste Feed Delivery Program. The velocity and pipeline pressure required for transfer increase as the sizes of the particles in the waste increase. The conservatism required to overcome the particle size uncertainty resulted in pipeline pressure estimates that exceed the design limits of the existing pipes and of those planned in current tank farm construction projects.

This document provides an improved understanding of the available particle size information used in performing the transfer system analysis. The goals of this document are to:

1. Perform a more detailed assessment, including statistical analysis, of available particlesize data for wastes in tanks at the Hanford Site

2. Provide refined, conservative, and more practical estimates of particle size for use in the transfer system analysis

3. Recommend further laboratory work and procedure changes to improve knowledge about the size of the particles in the wastes.

Measurements of the size of the particles of wastes in Hanford Site tanks have been made for many years. The results of 92 of those measurements are included in tables in this document. At least six different instrumental methods were used to obtain these measurements. The most recent measurements, obtained with a Horiba* Model LA-910 instrument, yielded results much higher than those obtained in the past. For all but one tank, the Horiba ${ }^{\mathrm{TM}}$ Model LA-910 yielded

\footnotetext{
"Horiba is a trademark of Horiba, Ltd., Kyoto, Japan.
} 
median particle sizes in the range of $46 \mu \mathrm{m}$ to $314 \mu \mathrm{m}$, while other instruments generally yield mean particle sizes less than $20 \mu \mathrm{m}$.

Factors have been examined to explain the differences in particle size measurement results obtained with the Horiba ${ }^{\text {TM }}$ Model LA-910 and the results obtained with other instruments. These factors include instrument design, analytical method, sampling, and sample pretreatment.

The low results of at least one instrument may be explained by instrument design. The Leeds \& Northrup Company Microtrac ${ }^{\dagger}$ Model UPA instrument has an upper bound of $6.5 \mu \mathrm{m}$, which causes particles with greater sizes to be undetected. Other instruments may not detect large particles because of insufficient stirring capabilities; the particles may simply be too heavy for the stirrer to bring them into the measuring region of the instrument. No aspects of instrument design can be cited that would lead to overestimation of particle sizes.

Analytical method and sample pretreatment may be responsible for a large share of the differences in the results of particle size measurements obtained with the Horiba ${ }^{\mathrm{TM}}$ Model LA-910 and the results obtained with other instruments. If the particulates are suspended in liquids of moderate to high ionic strength, agglomeration likely will occur. Agglomeration results in larger sized particles. All of the Horiba ${ }^{\mathrm{TM}}$ Model LA-910 measurements were taken in liquids with significant ionic strength; measurements made with other instruments were often taken in liquids of low ionic strength (i.e., water or water/glycerin mixtures).

The degree of turbulence during measurement also may affect the results of particle size distribution. Although agglomerates may be dispersed by sonication, mechanical mixing, or passage through pumps, the extent to which these actions have an effect is not well known. The rate of re-agglomeration has not been studied very much either; particle sizes in some measurements may be affected by a dynamic equilibrium between agglomerate disruption caused by stirring and re-agglomeration caused by the ionic strength of the suspending liquid.

\footnotetext{
${ }^{\dagger}$ Microtrac is a trademark of Leeds \& Northrup Company, North Wales, Pennsylvania.
} 
Recognition of agglomeration and de-agglomeration phenomena greatly complicates the identification of particle sizes that will be present during transfer. Although the particles present in a waste feed tank may be highly agglomerated, they will be subject to disruption by mixer pumps and a multistage turbine transfer pump before entering the transfer system piping. The extent to which the agglomerates will be diminished and the time required for re-formation of the agglomerates are not known. It cannot be assumed a priori that agglomerates will be disrupted into their component particles and remain de-agglomerated during transport.

Notwithstanding our indefinite understanding of these behaviors, a statistical analysis was performed to provide a bounding value for the median particle size suitable for use as a design basis for the waste transfer system. Because only trace amounts of solids are permitted in low-activity wastes, this statistical analysis was performed on data obtained for HLW only. Furthermore, to ensure that the outcome would provide a conservative design basis, the statistical analysis was restricted to measurements made with the Horiba ${ }^{\mathrm{TM}}$ Model LA-910, the instrument that provided the largest particle size results. These restrictions reduced the set of measurements in the statistical analysis to 21 measurements: 15 measurements from Tank 241-AW-103, 2 measurements from Tank 241-AZ-101, and 4 measurements from Tank 241-C-104.

The statistical analysis was conducted in three different ways: (1) using the analysis of variance method, (2) analyzing the mean distribution for each tank, and (3) analyzing the mean cumulative distribution for each tank. The results of each of these methods were approximately the same, in large part because of the relative similarity of the distributions from one sample to the next and from one tank to the next. The median particle size in these three HLW tanks is approximately $110 \mu \mathrm{m}$. It can be stated with $95 \%$ confidence that this value does not exceed $140 \mu \mathrm{m}$.

Furthermore, it can be assumed with some justification that the three tanks for which data are available are a random sample of the ten HLW tanks. This assumption permits calculation of a "tolerance limit" of approximately $275 \mu \mathrm{m}$ for the median particle size in each of the HLW tanks. The interpretation of this tolerance limit is that "we are $95 \%$ confident that the median particle size diameter will not exceed $275 \mu \mathrm{m}$ in at least $95 \%$ of the HLW tanks." 


\section{RPP-6427 REV 0}

This review of particle size measurements has focused understanding about the size of the particles in Hanford Site tank wastes, but the following issues remain unresolved:

- Unresolved difference between measurements made with the Horiba ${ }^{\mathrm{TM}}$ Model LA-910 and measurements made with other measuring instruments

- Uncertainty about the extent to which agglomerates in HLW slurries will be present during transport

- Uncertainty about the densities of the agglomerated particles.

If these uncertainties can be reduced, it may be possible to reduce costs for design, construction, and qualification of the waste feed delivery transfer system. A plan has been proposed to close these issues. The plan includes further literature assessments, additional laboratory work, and additional modeling of the transfer system.

\section{REFERENCES}

RPP-5346, 2000, Waste Feed Delivery Transfer System Analysis, Rev. 0, CH2M HILL Hanford Group, Inc., Richland, Washington. 


\section{CONTENTS}

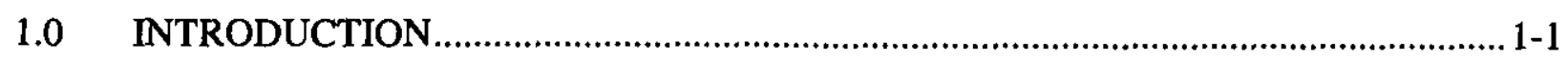

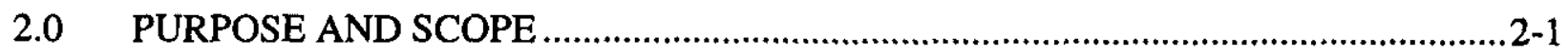

3.0 PRESENTATION AND REVIEW OF AVAILABLE DATA ........................................ 3-1

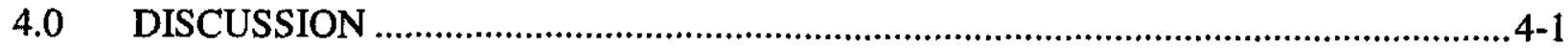

4.1 FACTORS THAT AFFECT PARTICLE SIZE MEASUREMENTS ……...........4-1

4.1.1 Instrument Design ................................................................................ 4-1

4.1.2 Analytical Method............................................................................ 4-4

4.1.3 Sample Source and Selection ............................................................4-6

4.1.4 Sample Pretreatment .......................................................................... 4-6

4.2 THE NATURE OF HIGH-LEVEL WASTE PARTICLES..................................4-7

4.3 STATISTICAL ANALYSIS OF PARTICLE SIZE DATA FOR HIGHLEVEL WASTE .......................................................................................4-8

4.3.1 Analysis of Mean Percentile Points ..................................................4-12

4.3.2 Analysis of Variance (ANOVA) ........................................................ 4-17

4.3.3 Analysis of Tank Cumulative Means ....................................................4-18

4.3.4 Summary of Statistical Analysis of Particle Size Data ........................... 4-18

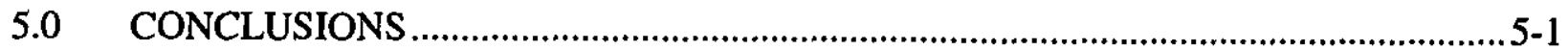

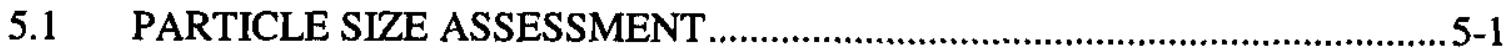

5.2 CALCULATION OF DESIGN BASIS VALUE ..............................................5-1

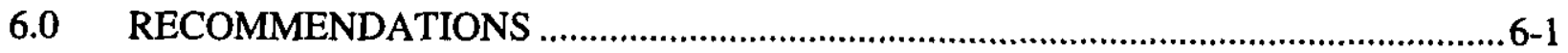

6.1 LITERATURE ASSESSMENTS .........................................................6-1

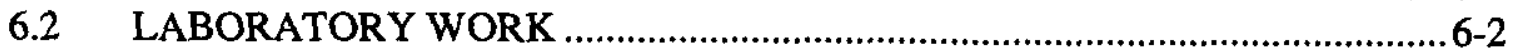

6.2.1 Validation of the Measurement Process....................................................6-2

6.2.2 Characterization of Waste Particles ........................................................6-2

6.3 MODELING OF THE TRANSFER SYSTEM …........................................... 6-3

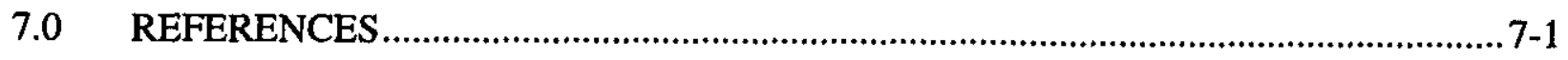

\section{APPENDIX}

A INDEPENDENT REVIEW OF CALCULATIONS.................................................. A-i 
RPP-6427 REV 0

FIGURES

Figure 4-1. Graphs of Particle Size Distributions Based on Means.

$4-14$

\section{TABLES}

Table 3-1. Particle Size Results for Samples from High-Level Waste Tanks

Table 3-2. Particle Size Results for Samples from Low-Activity Waste Tanks. .3-6

Table 3-3. Particle Size Results for Samples from Phase 2 Tanks.

Table 3-4. Comparison of Results of Measurements Using the Horiba ${ }^{\mathrm{TM}}$ Model LA-910 with Results of Measurements Using Other Instruments for Several Tanks.

Table 4-1. Advantages and Disadvantages of Particle Size Distribution Measurement Technologies.

Table 4-2. Major Waste Types in the High-Level Waste Tanks

Table 4-3. Particle Volume Distribution Data by Tank and Sample, the Maximum in Each is Shaded

Table 4-4. Mean Volume Percent in Bins

Table 4-5. Cumulative Particle Volume Distribution $4-15$

Table 4-6. Estimates of Quantiles, Based on Linear Interpolation, for Five Percentile Points

Table 4-7. Tolerance Limits and Confidence Limits on Mean Percentiles. 4-16

Table 4-8. Confidence Limits on Percentiles Based on Analysis of Variance Estimates of Means and Variance of Means.

Table 4-9. Cumulative Particle Volume Distribution Based on Tank Means 4-19

Table 4-10. Confidence Limits on Percentiles Based on Tank Cumulative Means. $.4-20$

Table 4-11. Comparison of Percentile Means, Tolerance Limits, and Confidence Limits $4-20$ 


\section{RPP-6427 REV 0}

\section{TERMS}

95/95 TL ANOVA

HLW

LAW

$\mu \mathrm{m}$

PSD

UL(95\%)

WFD upper limit of a one-sided 95/95 tolerance limit analysis of variance method

high-level waste

low-activity waste

micrometer

particle size distribution (measurement)

upper limit to a one-sided $95 \%$ confidence limit

Waste Feed Delivery (Program) 
RPP-6427 REV 0

This page intentionally left blank. 


\subsection{INTRODUCTION}

The purpose of the Waste Feed Delivery (WFD) Program of the River Protection Project is to deliver certain nuclear wastes stored in underground tanks at the Hanford Site to the planned Waste Treatment and Immobilization Plant. The wastes will be delivered by pipeline over distances exceeding $2000 \mathrm{~m}$ in some cases. The wastes to be fed to this new plant are classified as high-level waste (HLW) feeds and low-activity waste (LAW) feeds. The HLW feeds, containing the majority of the strontium and transuranic nuclides, have a much greater content of relatively insoluble solids and will be transferred as slurries. The LAW feeds will be transferred to the Waste Treatment and Immobilization Plant as liquids with minor amounts of entrained solids (HNF-SD-WM-SP-012).

As part of the WFD Program, an engineering analysis (RPP-5346) was performed to determine the adequacy of the waste transfer system (piping, pumps, valves, and fittings). For each transfer anticipated during Phase 1 of the River Protection Project, the velocity of the waste required to suspend and transport the solid fraction of the waste was determined. This velocity is known as the "critical velocity." The pipeline pressure required to achieve the critical velocity was determined also.

These analyses require knowledge of several characteristics of the waste, including the volume fraction of solids, densities of the liquid and solids, viscosity of the liquid, and particle size of the solids. Although none of these quantities are known with a great deal of precision, the uncertainty in the particle size distribution (PSD) data affected the calculations most seriously, resulting in estimates for required pipeline pressures that greatly exceed the design limits.

To support the waste transfer analysis, it was necessary to clarify some of the uncertainty associated with PSD. A plan for addressed this issue was documented in Work Plan to Reduce Uncertainty of Particle Size Estimates (Jewett 2000), and the work was approved. This document reports the results of that work. 
RPP-6427 REV 0

This page intentionally left blank. 


\section{RPP-6427 REV 0}

\subsection{PURPOSE AND SCOPE}

The purpose of this document is to provide an improved understanding of the available particle size information to support the waste transfer system analysis. The goals of this document are to:

1. Perform a more detailed assessment, including statistical analysis, of available laboratory data for PSD in the wastes

2. Provide a refined, conservative, and more practical estimate of particle size for use in the transfer system analysis

3. Recommend further laboratory work and procedure changes to improve knowledge about the size of the particles in the wastes.

The work in this document was approved in Jewett (2000). 


\section{RPP-6427 REV 0}

This page intentionally left blank. 


\subsection{PRESENTATION AND REVIEW OF AVAILABLE DATA}

Tables 3-1, 3-2, and 3-3 present the results of particle size measurements obtained for the wastes. Table 3-1 contains data for tanks selected as HLW feed tanks for Phase 1 of the River Protection Project; Table 3-2 contains data for Phase 1 LAW feed tanks; and Table 3-3 contains data for Phase 2 feed tanks. All statistics listed in these tables are reported in micrometers $(\mu \mathrm{m})$ and are based on distributions weighted according to the volumes of the particles.

The particle size data were obtained from documents and internal letters produced by U.S. Department of Energy contractors over a period of more than a decade; these reference sources are cited in the tables. At least six different types of instruments were used to collect the data; the type of instrument used varied according to the year and laboratory in which the measurements were made. At the beginning of this period, the 222-S Laboratory at the Hanford Site was using an instrument manufactured by HIAC/ROYCO ${ }^{1}$; over the years, this was replaced by the Brinkmann ${ }^{2}$ Model 2010, then by the Horiba ${ }^{3}$ Model LA-910. The various researchers at the Pacific Northwest National Laboratory used the Brinkmann ${ }^{\mathrm{TM}}$ Model 2010 and three different models from the Leeds \& Northrup Company Microtrac ${ }^{4}$ line. The differences in designs among these instruments are discussed in Section 4.1.1.

Of the 10 tanks selected as sources for HLW feed (HNF-SD-WM-SP-012), particle size data were available for 8 tanks. No measurements were found for HLW Tanks 241-AY-102 and 241-AW-104. Of the 18 tanks selected as sources for LAW feed, particle size data are available for 5 tanks. The number of Phase 1 LAW tanks for which no particle size data are available far exceeds the number of HLW tanks for which no such data are available. However, the LAW data are of less concern because very little solid material is to be transferred in the LAW.

The data in Tables 3-1, 3-2, and 3-3 reveal that the particle size measurements obtained with the Horiba $^{\mathrm{TM}}$ Model LA-910 instrument generally are substantially larger than the results of measurements made using the other instruments. With the exception of Tank 241-U-109, the median particle sizes measured with the Horiba ${ }^{\mathrm{TM}}$ Model LA-910 ranged from $46 \mu \mathrm{m}$ to $314 \mu \mathrm{m}$. (The medians for two samples from Tank 241-U-109 were 13 and $17 \mu \mathrm{m}$.) However, median particle sizes obtained with the other instruments exceeded $20 \mu \mathrm{m}$ for only one tank. Values ranging from $11.5 \mu \mathrm{m}$ to $46 \mu \mathrm{m}$ were obtained on samples from Tank 241-BY-104 using the Brinkmann ${ }^{\mathrm{TM}}$ Model 2010 instrument. In addition, a mean value of $26 \mu \mathrm{m}$ was found for a sample from Tank 241-SX-108 using the Microtrac ${ }^{\mathrm{TM}}$ Model X-100, and distribution means of $48 \mu \mathrm{m}$ and $129 \mu \mathrm{m}$ were found in two measurements of samples from Tank 241-S-111. The instrument used for obtaining the measurements in Tank 241-S-111 was not specified.

\footnotetext{
${ }^{1}$ HIAC/ROYCO is a trademark of Pacific Scientific Company, Anaheim, California.

${ }^{2}$ Brinkmann is a trademark of Brinkmann Instruments, Inc., Westbury, New York.

${ }^{3}$ Horiba is a trademark of Horiba, Ltd., Kyoto, Japan.

${ }^{4}$ Microtrac is a trademark of Leeds \& Northrup Company, North Wales, Pennsylvania.
} 


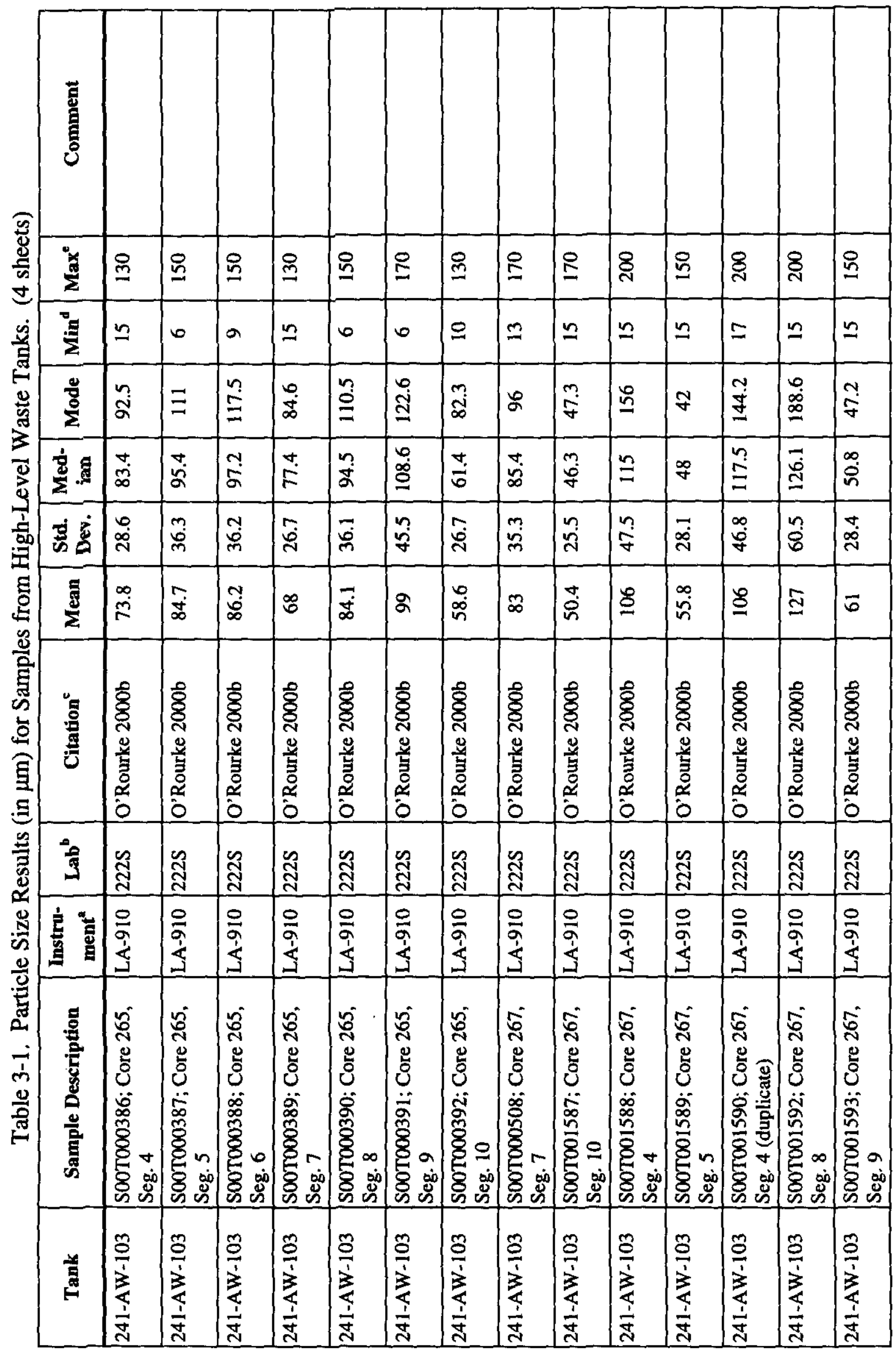


RPP-6427 REV 0

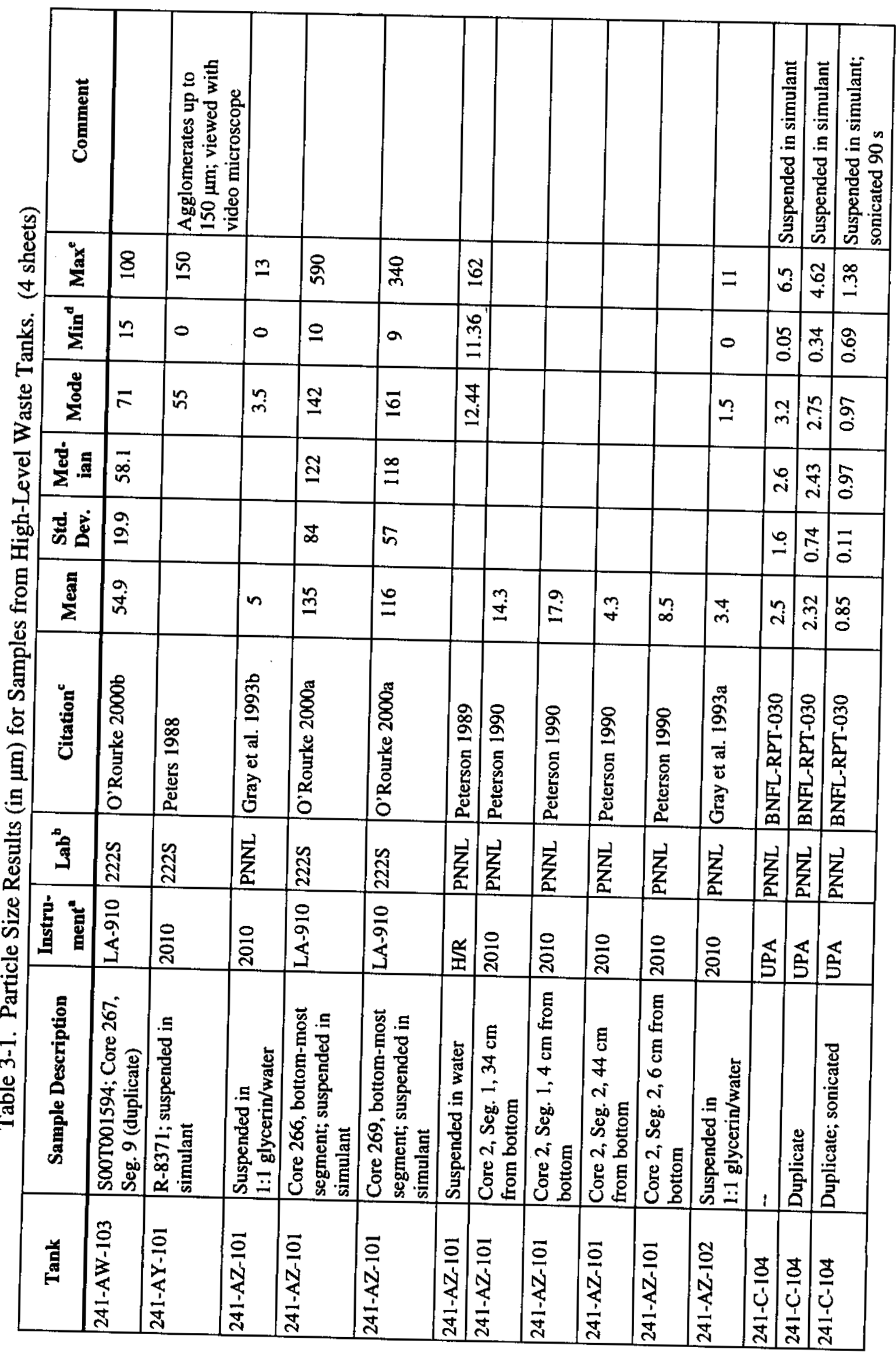


RPP-6427 REV 0

\begin{tabular}{|c|c|c|c|c|c|c|c|c|c|c|c|c|c|}
\hline 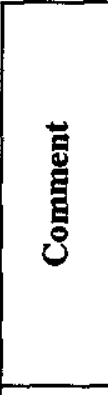 & 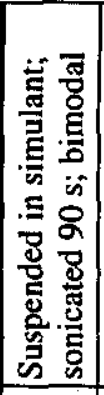 & 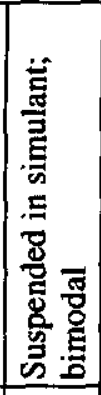 & 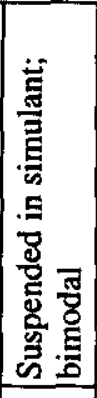 & 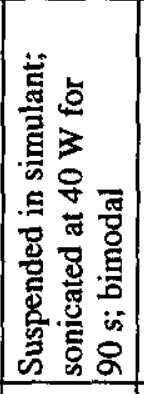 & 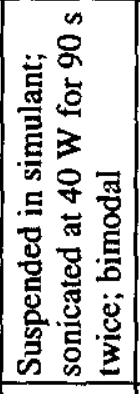 & 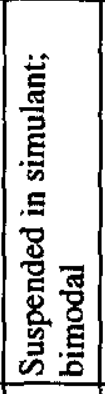 & 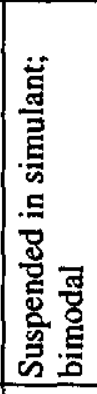 & 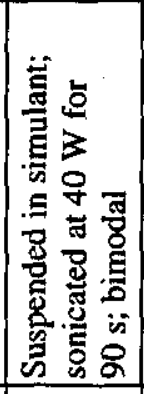 & 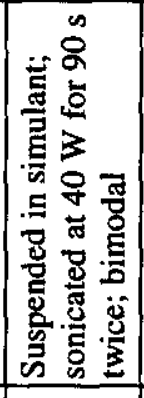 & & & & \\
\hline 离 & $\begin{array}{l}\stackrel{\partial}{\rho} \\
\text { ले }\end{array}$ & 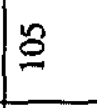 & $\infty$ & $\infty$ & $\infty$ & ก & N & $\approx$ & $N$ & ర్లి & ৪্ণ & $\tilde{\Omega}$ & $\cong$ \\
\hline 豆 & ले & ণ্ & $\stackrel{\mathbb{্}}{\stackrel{0}{0}}$ & ஸु & O & ঙ্ণ & $\stackrel{\sim}{\sim}$ & 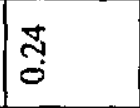 & ণ్ & 0 & 0 & $n$ & $\stackrel{\circ}{0}$ \\
\hline$\stackrel{8}{8}$ & $\stackrel{n}{i}$ & $\stackrel{+}{=}$ & 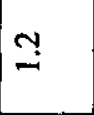 & $\cong$ & $\stackrel{\sim}{\sim}$ & $\stackrel{+}{-}$ & $\stackrel{\Upsilon}{-}$ & & & 艺 & $\stackrel{\mathscr{I}}{\cong}$ & $\stackrel{N}{\mathcal{N}}$ & $\stackrel{\infty}{ᄋ}$ \\
\hline 总 & $\stackrel{n}{-}$ & $\stackrel{\emptyset}{m}$ & $\underset{r}{\dot{r}}$ & $\stackrel{m}{m}$ & $m$ & $\stackrel{n}{n}$ & $\stackrel{\infty}{\infty}$ & $\dot{m}$ & $\vec{m}$ & $\equiv$ & 8 & $\stackrel{\infty}{=}$ & $\approx$ \\
\hline 密 & $\stackrel{8}{\varnothing}$ & $=$ & $\stackrel{m}{0}$ & $\stackrel{\circ}{\circ}$ & $\stackrel{9}{\infty}$ & $a^{\infty}$ & $a$ & $\alpha^{\infty}$ & ه̆ & $F$ & 운 & $\alpha$ & P \\
\hline$\stackrel{g}{\mathrm{~g}}$ & $\stackrel{\curvearrowleft}{\longleftarrow}$ & $\stackrel{0}{\circ}$ & 2 & $\vec{a}$ & $\underset{\infty}{n}$ & $T_{\infty}$ & $\infty$ & $\underset{\infty}{-}$ & $\underset{\infty}{m}$ & 曲 & 8 & \pm & 8 \\
\hline ل & 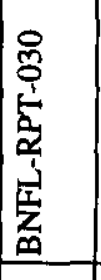 & 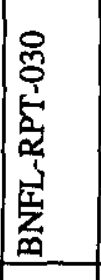 & 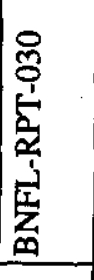 & 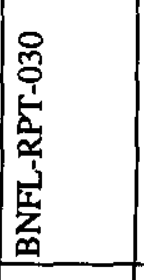 & 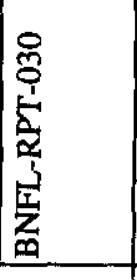 & 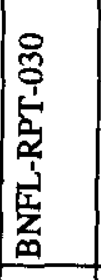 & 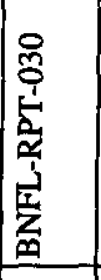 & 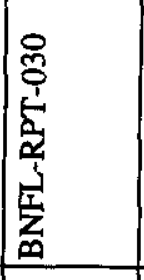 & 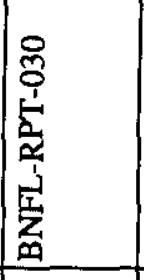 & 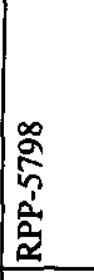 & 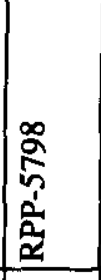 & 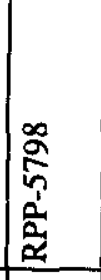 & 离 \\
\hline คै & 党 & 空 & 叓 & 空 & 空 & 点 & 帘 & 总 & 悹 & స్心 & స్తి & స్ & స్తి \\
\hline 悹 & 芯 & $\frac{8}{x}$ & $\frac{8}{x}$ & $\frac{8}{x}$ & $\frac{8}{x}$ & $\frac{8}{x}$ & $\frac{8}{x}$ & $\frac{8}{x}$ & $\frac{8}{x}$ & 象 & $\stackrel{0}{\frac{9}{9}}$ & $\stackrel{0}{a}$ & 옹 \\
\hline 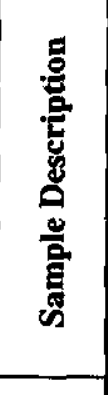 & 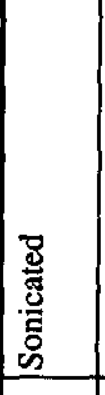 & 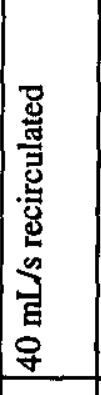 & 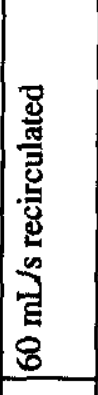 & 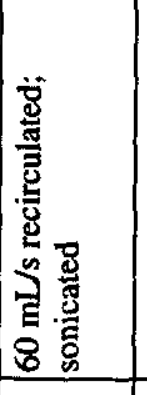 & 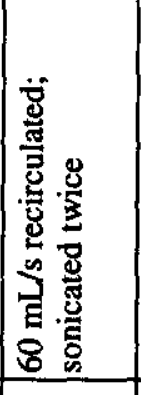 & 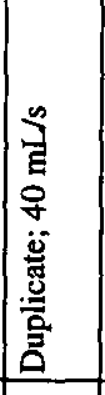 & 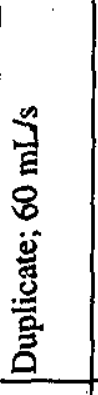 & 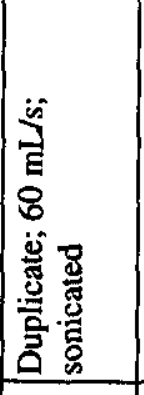 & 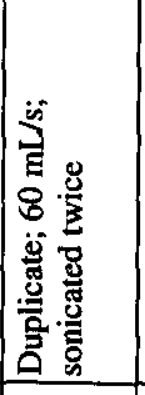 & 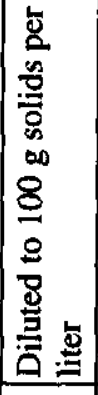 & 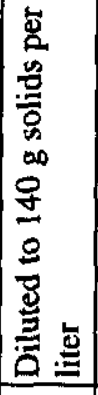 & 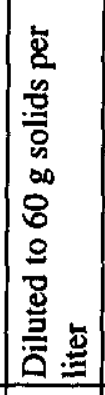 & 胥 \\
\hline 麊 & $\frac{\text { 号 }}{3}$ & $\frac{⿱}{0}$ & $\frac{⿱ 艹}{0}$ & $\frac{⿱}{d}$ & $\frac{d}{d}$ & $\frac{⿱}{0}$ & 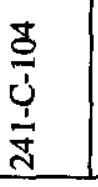 & 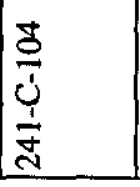 & 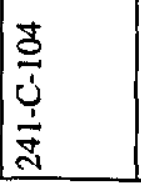 & 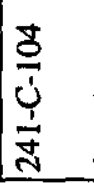 & 薄 & 志 & $\frac{⿱}{0}$ \\
\hline
\end{tabular}




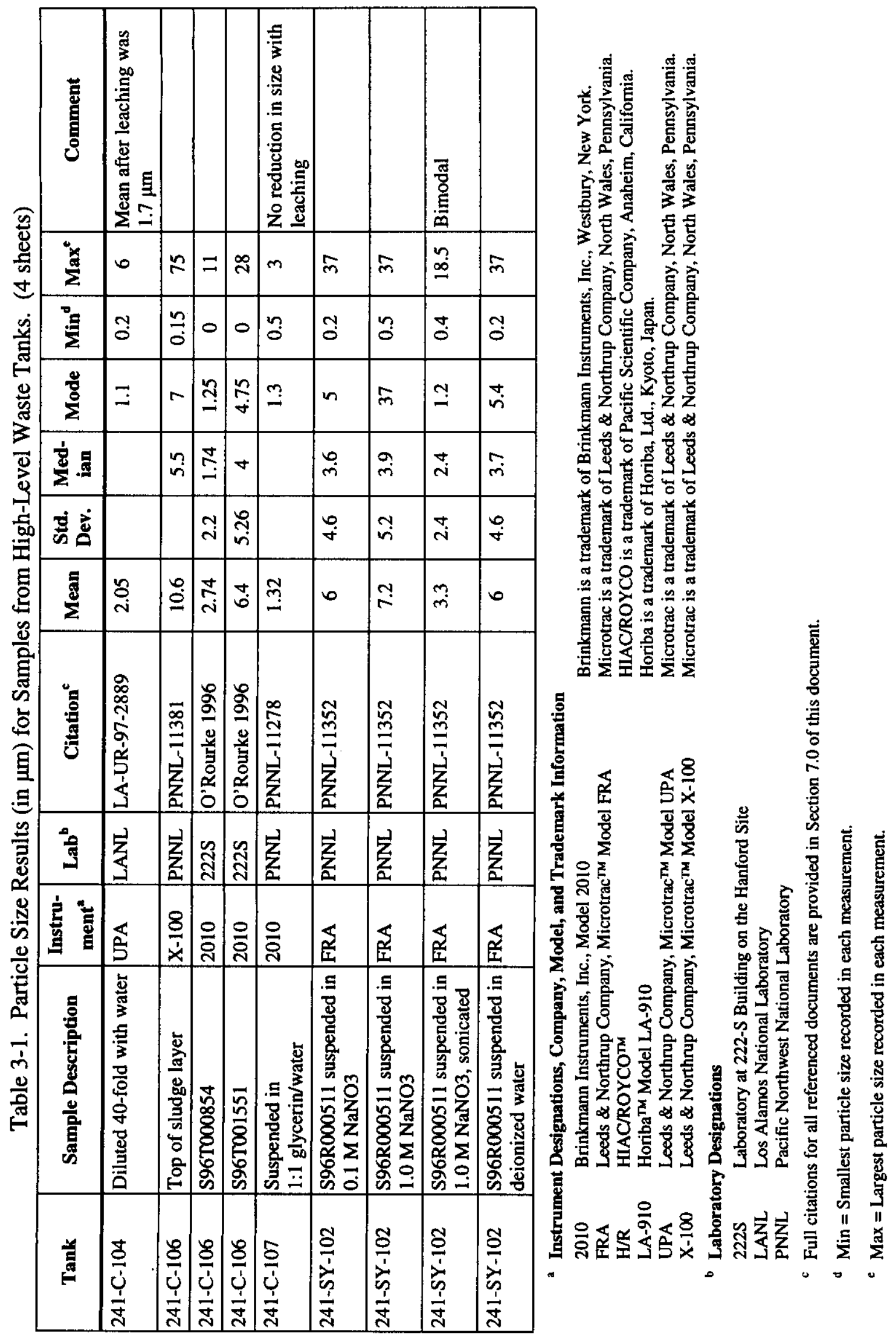




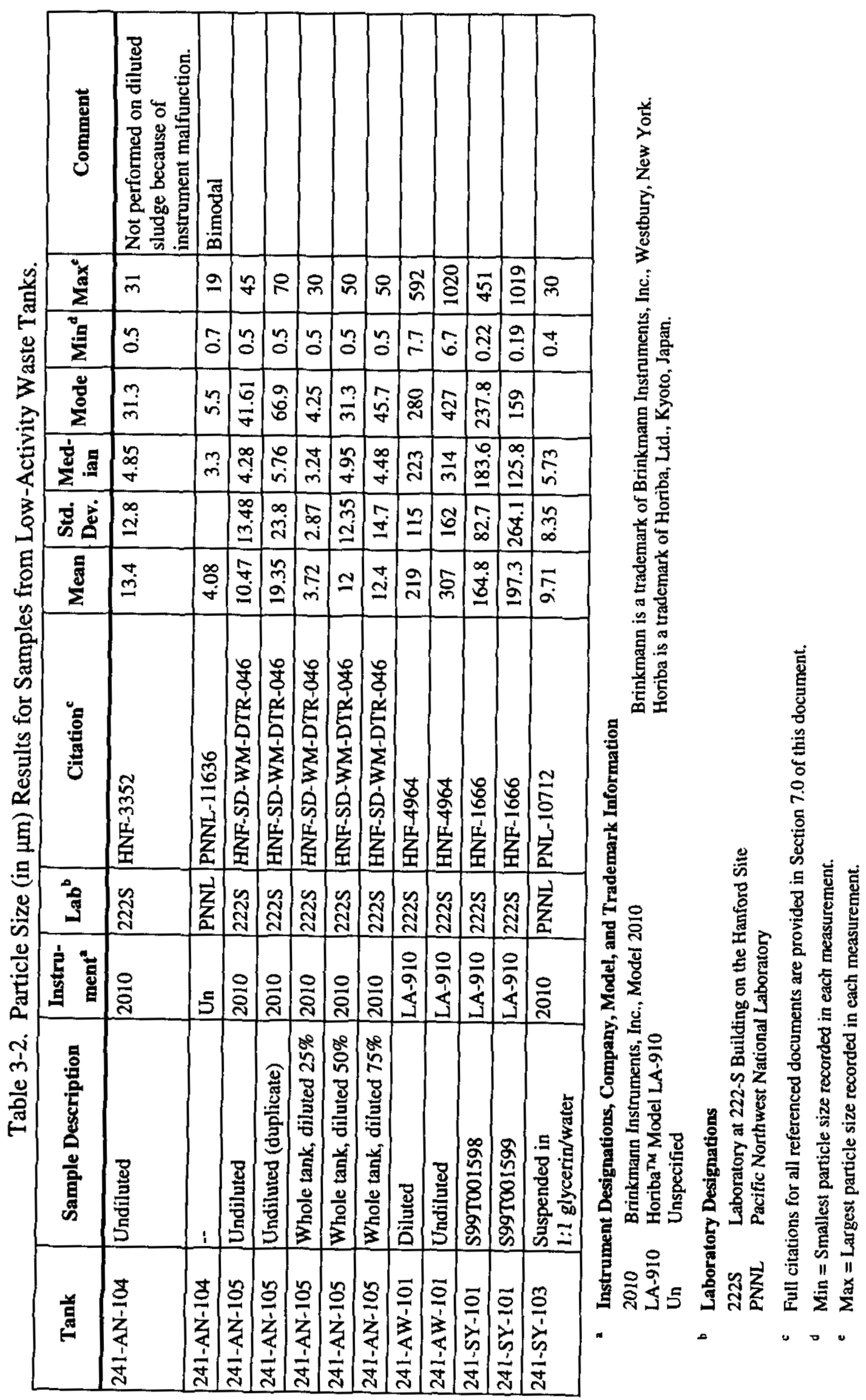




\section{RPP-6427 REV 0}

\begin{tabular}{|c|c|c|c|c|c|c|c|c|c|c|c|c|}
\hline $\begin{array}{l}\vec{E} \\
\ddot{E} \\
\dot{E}\end{array}$ & 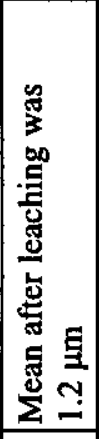 & & 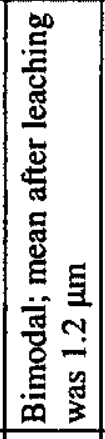 & & 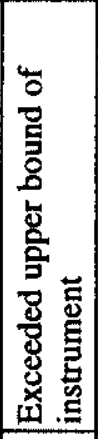 & 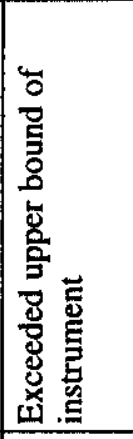 & & 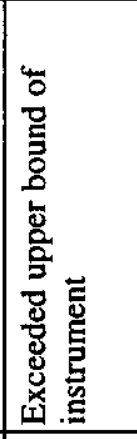 & 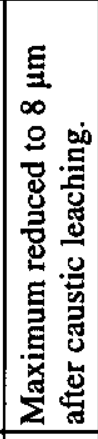 & & & \\
\hline$\stackrel{x}{\Sigma}$ & 0 & a & 0 & $m$ & $\stackrel{n}{2}$ & 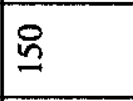 & 을 & $\stackrel{\circ}{2}$ & $\approx$ & & ले & \\
\hline 䜿 & ָั & ণ্ & $\check{0}$ & $\dot{0}$ & - & ñ & $n$ & - & $\stackrel{\Delta}{0}$ & & $\stackrel{\sim}{0}$ & \\
\hline$\stackrel{8}{g}$ & $\forall$ & & 年 & & $\approx$ & $\stackrel{\sim}{*}$ & $\infty$ & $\stackrel{\infty}{\varrho}$ & a & & & \\
\hline 总 & & $\vec{m}$ & & $\stackrel{\sim}{*}$ & $\underset{m}{\infty}$ & 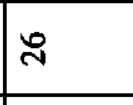 & $\dddot{n}$ & \& & $\infty$ & & & \\
\hline 岀 岕 & & $\stackrel{\infty}{=}$ & & $\begin{array}{l}\infty \\
\dot{m}\end{array}$ & 요 & 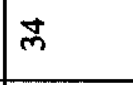 & 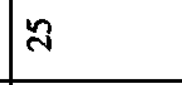 & s & & & & \\
\hline$\stackrel{\Sigma}{E}$ & 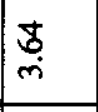 & $\underset{m}{\mathscr{0}}$ & $\cong$ & in & P & 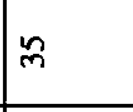 & $\ddot{\sim}$ & 芯 & $\bar{n}$ & \%̊ & ڤ̆ & \\
\hline 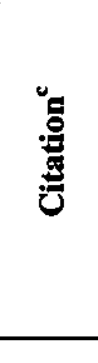 & 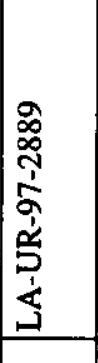 & $\frac{N}{\vdots}$ & 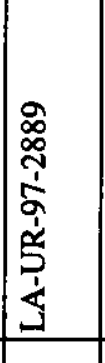 & 站 & 㝵 & 是 & 是 & 是 & 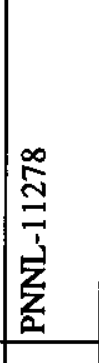 & 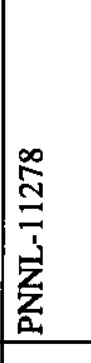 & 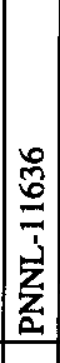 & 紊 \\
\hline คै & 㹸 & 空 & 岁 & 空 & ส্ণি & สิ & สุ & స్ & 玆 & 空 & 帘 & 空 \\
\hline 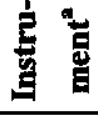 & 占 & 옹 & 方 & 옹 & 옹 & 음 & 융 & 융 & $\frac{8}{\dot{x}}$ & $\frac{8}{\dot{x}}$ & 5 & $\frac{8}{x}$ \\
\hline 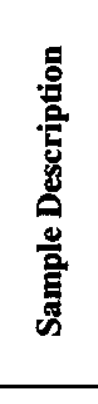 & 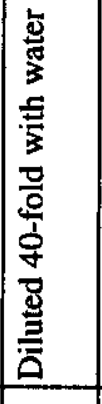 & 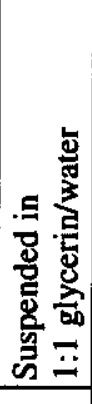 & 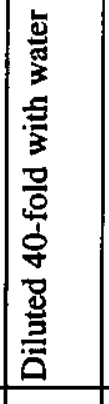 & 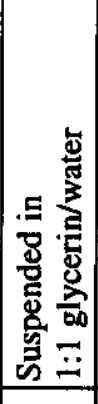 & 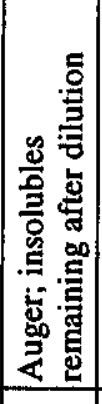 & 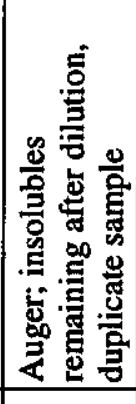 & 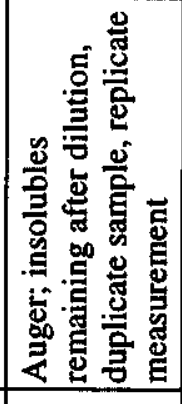 & 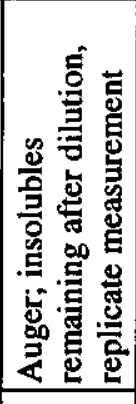 & 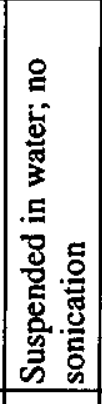 & 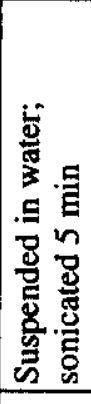 & 1 & 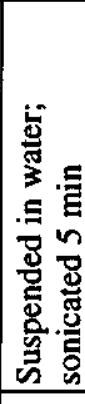 \\
\hline 葛 & 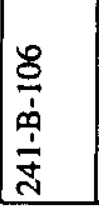 & 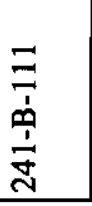 & 竞 & 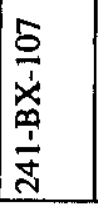 & 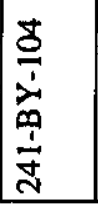 & 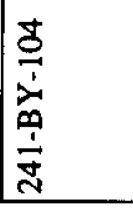 & 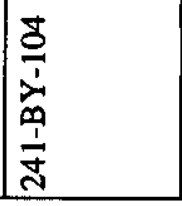 & 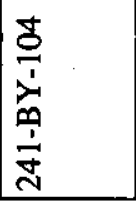 & 竞 & 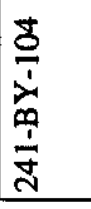 & 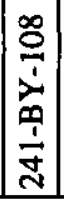 & 窝 \\
\hline
\end{tabular}




\begin{tabular}{|c|c|c|c|c|c|c|c|c|c|c|c|c|c|c|}
\hline $\begin{array}{l}\text { 总 } \\
\text { 昜 }\end{array}$ & 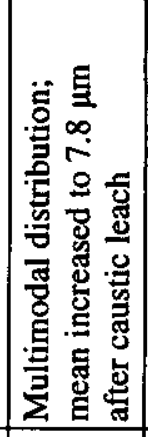 & & 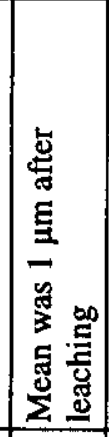 & & & 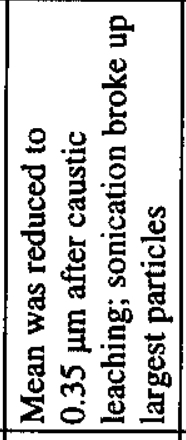 & & & & & & 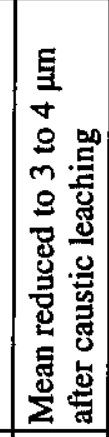 & 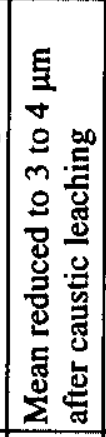 & 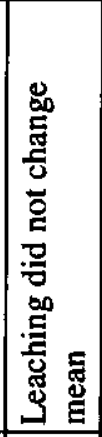 \\
\hline$\sum^{\circ}$ & q & $\sim$ & 0 & $\stackrel{\infty}{m}$ & ஜి & 8 & ষ্ল & \& & 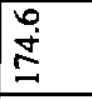 & $\bar{y}$ & 气̃ & & & 0 \\
\hline 蛋 & 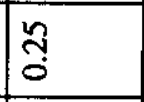 & $\stackrel{\nabla}{0}$ & $\tilde{\sigma}$ & : & 芯 & $\stackrel{n}{m}$ & $\stackrel{a}{0}$ & - & 2 & $\stackrel{0}{=}$ & $\overline{0}$ & & & ָุ \\
\hline$\frac{\tilde{g}}{\tilde{g}}$ & 9 & & $\stackrel{\infty}{-}$ & & & $\tilde{N}$ & & & $\begin{array}{l}0 \\
\stackrel{0}{\circ} \\
\end{array}$ & ڤั̀ & $\frac{0}{2}$ & & & 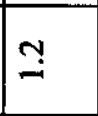 \\
\hline 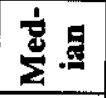 & $N$ & $\stackrel{8}{-}$ & & & $\stackrel{\tilde{m}}{n}$ & $\dddot{n}$ & & & 苞 & \& & à & & & \\
\hline के & & $\stackrel{2}{3}$ & & & $\stackrel{\text { ஸ্ }}{-}$ & & & & $\begin{array}{l}0 \\
0 \\
0\end{array}$ & 2 & $\stackrel{\infty}{\infty}$ & & & \\
\hline 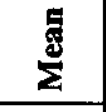 & $\stackrel{\infty}{+}$ & $\stackrel{8}{8}$ & $\stackrel{\circ}{\text { i }}$ & $\mid \begin{array}{l}\infty \\
\dot{0} \\
\dot{0}\end{array}$ & $\stackrel{\infty}{\stackrel{\infty}{\sim}}$ & $\stackrel{\infty}{\stackrel{\infty}{\Xi}}$ & $\frac{2}{8}$ & $\stackrel{2}{\beth}$ & 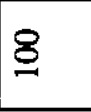 & 离 & बू' & দ̀̀ं & $\stackrel{\infty}{\stackrel{\infty}{\varrho}}$ & 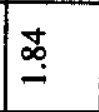 \\
\hline 䓂 & 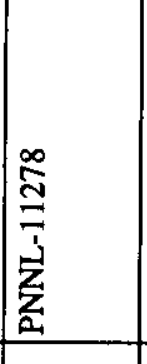 & 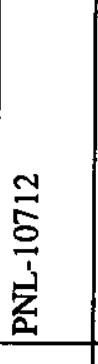 & 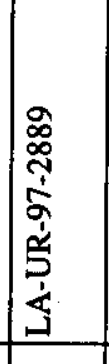 & 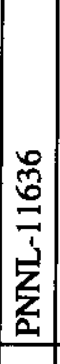 & 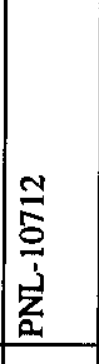 & 产 & 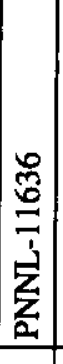 & 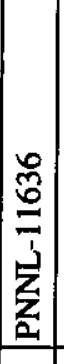 & $\frac{\text { 吾 }}{\frac{1}{\mathbf{3}}}$ & 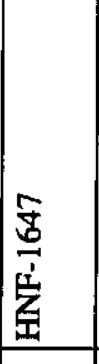 & 喜 & 总 & 意 & 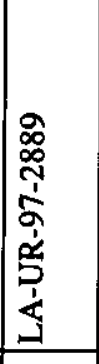 \\
\hline คै & 紡 & 空 & 案 & 空 & 㚆 & 忘 & 文 & 空 & స్ & స్తి & స్ & 豙 & 总 & 宗 \\
\hline 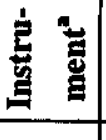 & $\frac{8}{x}$ & 융 & 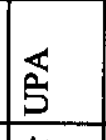 & $\Xi$ & 옥 & $\frac{8}{x}$ & s & 5 & $\frac{1}{4}$ & \begin{tabular}{|l|l}
9 \\
\\
\end{tabular} & $\frac{0}{9}$ & $\frac{8}{x}$ & $\frac{8}{x}$ & S \\
\hline 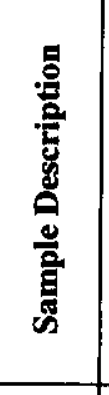 & 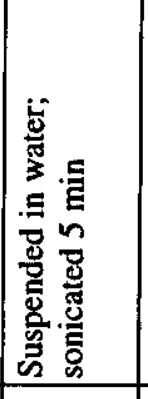 & 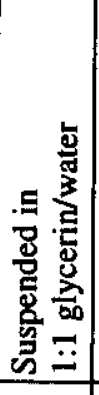 & 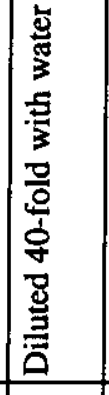 & 1 & 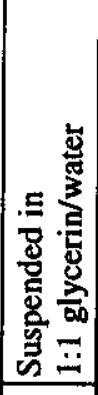 & 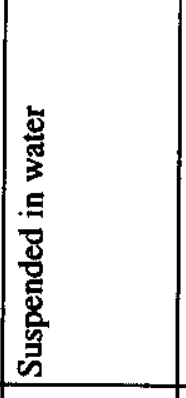 & 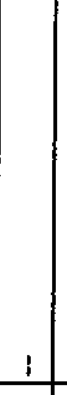 & 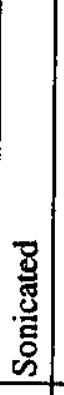 & 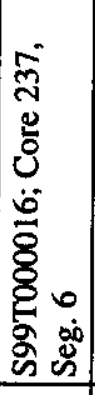 & 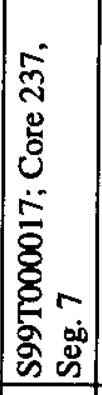 & 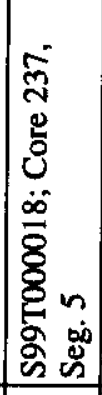 & 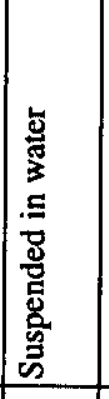 & 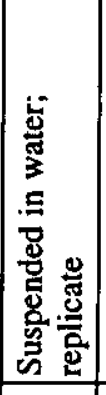 & 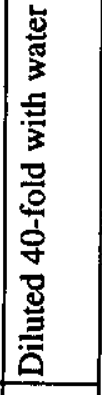 \\
\hline 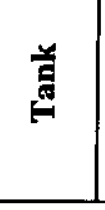 & 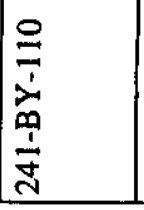 & 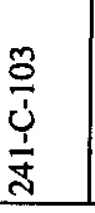 & $\frac{n}{\stackrel{3}{3}}$ & $\frac{\bar{\sigma}}{\frac{1}{b}}$ & 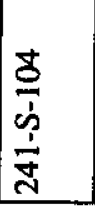 & 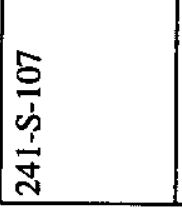 & 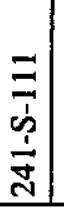 & 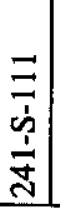 & $\begin{array}{l}\equiv \\
\frac{\dot{b}}{\vec{j}}\end{array}$ & 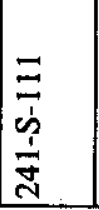 & $\frac{\Xi}{\grave{d}}$ & 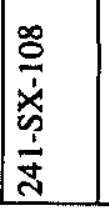 & 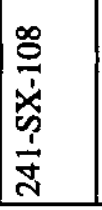 & 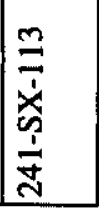 \\
\hline
\end{tabular}




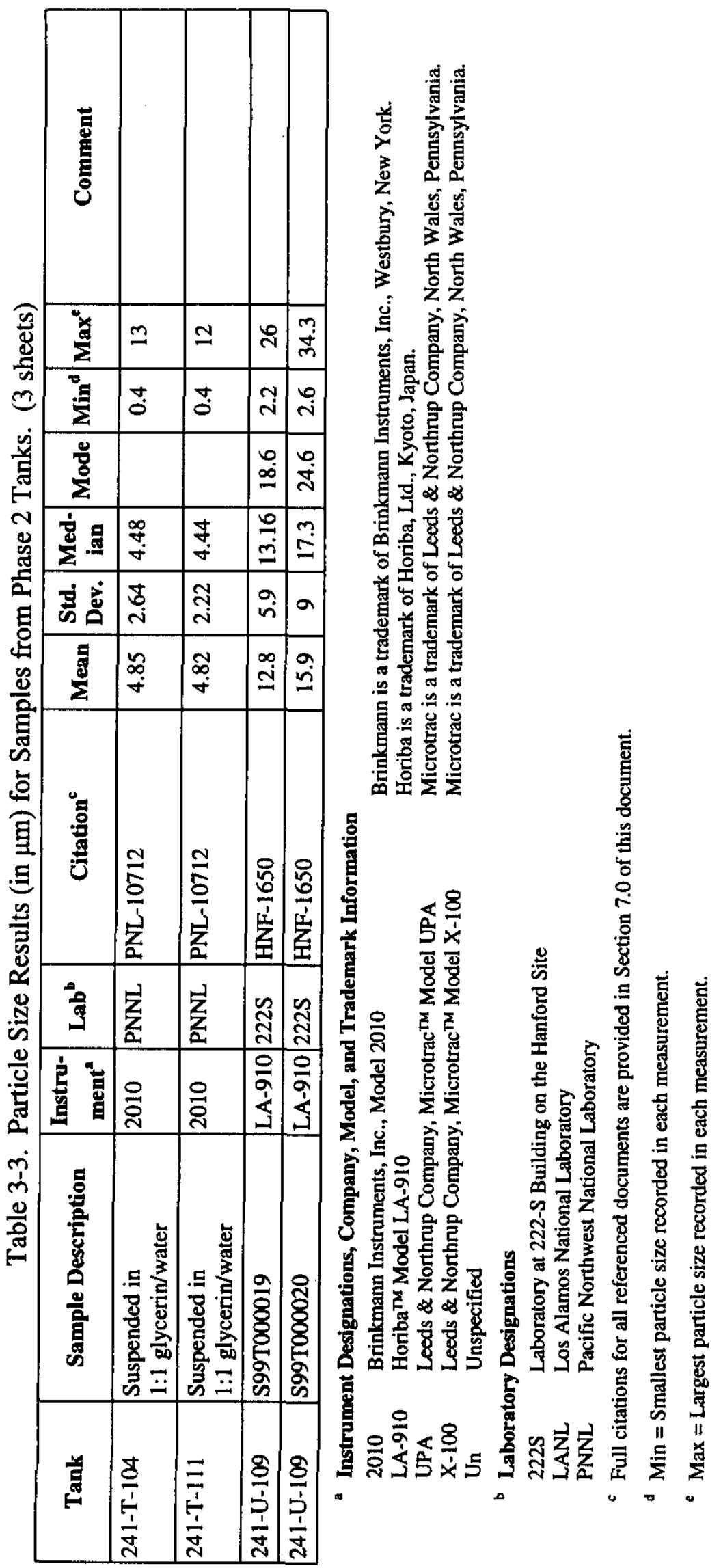


The difference between results obtained using the Horiba ${ }^{\mathrm{TM}}$ Model LA-910 and the other instruments could be circumstantial (i.e., the tanks tested with the Horiba ${ }^{\mathrm{TM}}$ Model LA-910 actually do have larger particles than those tested with the other instruments), but the chances of this occurring are unlikely. To demonstrate the differences more rigorously, the instruments should be compared using samples from the same tanks. Measurements were obtained using the Horiba ${ }^{\mathrm{TM}}$ Model LA-910 and at least one other instrument on samples from Tanks 241-S-111, 241-AZ-101, and 241-C-104. Results of these measurements are provided in Table 3-4. Measurements taken using the Horiba ${ }^{\mathrm{TM}}$ Model LA-910 were substantially higher in every case than measurements taken with other instruments.

Table 3-4. Comparison of Results of Measurements Using the Horiba ${ }^{\mathrm{TM}}$ Model LA-910 with Results of Measurements Using Other Instruments for Several Tanks.

\begin{tabular}{|c|c|c|c|}
\hline \multirow[b]{2}{*}{ Tank } & \multirow{2}{*}{$\begin{array}{c}\text { Results using } \\
\text { Horiba }^{\mathbf{a}} \\
\text { Model LA-910 }^{\left.\text {(mean, }{ }^{\mathbf{b}} \boldsymbol{\mu m}\right)}\end{array}$} & \multicolumn{2}{|c|}{ Other Instruments } \\
\hline & & Make and Model ${ }^{\mathrm{c}}$ & $\begin{array}{c}\text { Results } \\
\left(\text { mean, }{ }^{b} \mu \mathrm{m}\right)\end{array}$ \\
\hline 241-S111 & $168,92,100$ & Unspecified & 47.7 \\
\hline 241-AZ-101 & 135,116 & Brinkmann Model 2010 & $\begin{array}{l}5,14.3,17.9,4.3, \\
8.5\end{array}$ \\
\hline \multirow[t]{2}{*}{ 241-C-104 } & \multirow[t]{2}{*}{69} & Microtrac ${ }^{\mathrm{TM}}$ Model UPA & $2.5,1.56,2.32,0.85$ \\
\hline & & Microtrac $^{\text {TM }}$ Model X-100 & $\begin{array}{l}10.6,9.7,9.1,8.5 \\
8.7,8.8,8.7,8.3\end{array}$ \\
\hline
\end{tabular}

"Horiba is a trademark of Horiba, Ltd., Kyoto, Japan.

${ }^{b}$ Means are tabulated because they were reported for most measurements. Results are not listed in any particular order.

${ }^{\mathrm{c}}$ Brinkmann is a trademark of Brinkmann Instruments, Inc., Westbury, New York. Microtrac is a trademark of Leeds \& Northrup Company, North Wales, Pennsylvania. 


\subsection{DISCUSSION}

In Section 4.3, the measurements listed in Tables 3-1, 3-2, and 3-3 will be used to develop a bounding value for the median particle size that may be used as a design basis for the WFD transfer system. The first step in developing this value is to select appropriate measurements from the tables. Before this selection can be made, however, some knowledge is needed about particle size measurement and the properties of particles in the slurries. Factors affecting particle size measurement are discussed in Section 4.1; information about the nature of sludge particles is presented in Section 4.2.

\subsection{FACTORS THAT AFFECT PARTICLE SIZE MEASUREMENTS}

This section addresses the factors that affect the results of particle size measurements. As stated previously, the results of particle size measurements obtained using the Horiba ${ }^{\text {TM Model LA-910 }}$ are inconsistent with the results of measurements taken using other instruments. If the measurements taken using the Horiba ${ }^{\mathrm{TM}}$ Model LA-910 were not taken into account, it would be reasonable to assume that the mean particle sizes in the waste tanks were generally less than $50 \mu \mathrm{m}$, and usually well below this value. If that were the case, the transfer system model (RPP-5346) would show that planned transfers could be accomplished within current system design parameters. It might be argued that the measurements obtained using the Horiba ${ }^{\mathrm{TM}}$ Model LA-910 must be in error because they disagree with the particle size measurements made with a number of other instruments. However there are a number of other possibilities to consider.

To assess the adequacy of a measurement, there are at least four different factors that should be considered: the instrument design, the manner in which the instrument is used (the analytical method), the source and selection of the samples, and the pretreatment of the sample.

To determine if there is a sound basis for accepting the Horiba ${ }^{\text {TM }}$ Model LA-810 particle size measurements, each of these factors must be considered. The following subsections address these factors.

\subsubsection{Instrument Design}

The instruments used to obtain the particle size measurements shown in Tables 3-1, 3-2, and 3-3 employed a variety of measurement technologies. The HIAC/ROYCO ${ }^{\mathrm{TM}}$ instrument measured particle sizes as a function of the amount of light that is blocked as the liquid-borne particles are forced through a hole illuminated with a high-intensity light beam (Allen 1997, pp. 352-3). The Brinkmann ${ }^{\mathrm{TM}}$ Model 2010 instrument scanned the sample rapidly with a highly focused laser beam. As the beam encounters a particle, the beam is blocked, and the particle size is related to the time of blockage (Allen 1997, p. 363). The Microtrac ${ }^{\mathrm{rM}}$ Model UPA used photon-correlation spectroscopy, which is based on the Doppler shift of scattered light resulting from Brownian movement of the particles (Allen 1997, pp. 426-435). 
The Horiba ${ }^{\mathrm{TM}}$ Model LA-910 and the Microtrac ${ }^{\mathrm{TM}}$ Model X-100 are based on the Mie theory of light scattering (Allen 1997, pp. 404-406). This effect produces a pattern of light intensity compared against the scattering angle. Light is scattered from large particles at only very small angles, whereas smaller particles tend to scatter light in all directions. The light-scattering patterns are quite complex, especially at small, forward-scattering angles. The light measurements taken at a number of different angles are converted into PSDs using proprietary numerical methods.

The most important feature of a particle size instrument is the range of particle sizes that it can detect and measure. The different technologies used and the ways in which the technologies are implemented result in instruments with various measuring ranges. These ranges are compared in Table 4-1, along with advantages and disadvantages of the various technologies. ${ }^{5}$ It is always necessary to consider whether the measuring range of the instrument is adequate for the sample. The measurements obtained in BNFL-RPT-030 show clearly that the range of the Microtrac Model UPA is not adequate for HLW. This document shows measurements made with the Microtrac ${ }^{\mathrm{TM}}$ Model X-100 that far exceed the $6 \mu \mathrm{m}$ measuring range of the Microtrac ${ }^{\mathrm{TM}}$ Model UPA.

Because the Horiba ${ }^{\text {TM }}$ Model LA-910 particle size measurements are so different from measurements taken using other instruments, some inquiry has been made into sources of error specific to light-scattering instruments. In these instruments, errors may be introduced if the actual particle properties differ from those assumed in the Mie theory. These properties include refractive index, surface roughness, and sphericity.

The Mie theory in general requires that both the real and imaginary components of the refractive index of the particles and the liquid be known. However, for particles larger than the wavelength of the light being diffracted, the theory devolves to the Fraunhofer approximation, which is independent of the refractive index (Allen 1997, p. 405). Because the measured sizes of waste particles substantially exceed the $633 \mathrm{~nm}$ wavelength of the laser light used in the Horiba ${ }^{\mathrm{TM}}$ Model LA-910, the refractive index should have no effect. The Mie theory assumes that the particles are smooth and spherical. The presence of rough particles will cause the distribution to be weighted toward smaller particles, and nonspherical particles cause only a broadening of the distribution consistent with the averaging of the particle dimensions (Allen 1997, p. 405).

Errors may be introduced into calculations of particle size from scattered-light data depending on the number of detectors used, how well the detectors are placed to receive the scattered light, and the proprietary details of the calculations. For instance, if the instrument design is not appropriate for very small particles, the results may contain false peaks in the PSDs (Bott and Hart 1991). The optics of the Horiba ${ }^{\mathrm{TM}}$ Model LA-910 are designed to measure particles with diameters ranging from $0.02 \mu \mathrm{m}$ to $1020 \mu \mathrm{m}$. This range exceeds that of any other instrument discussed in this document.

\footnotetext{
${ }^{5}$ Table 4-1 includes two technologies-microscopic observation (with electron beams or visible light) and sievingthat were not used to obtain the data reported in Tables 3-1, 3-2, and 3-3 but have been suggested for measuring PSD in highly radioactive wastes. Settling also has been suggested as an alternative way to characterize particle sizes, but a settling test ordinarily results only in an observation of the settling rate of the slowest particles and cannot determine an actual distribution of particle sizes.
} 
Table 4-1. Advantages and Disadvantages of Particle Size Distribution Measurement Technologies.

\begin{tabular}{|c|c|c|c|c|}
\hline Technology & Instrument" & $\begin{array}{l}\text { Measurement } \\
\text { range }(\mu \mathrm{m})\end{array}$ & Advantages & Disadvantages \\
\hline $\begin{array}{l}\text { Light blocking/ } \\
\text { hole }\end{array}$ & HIAC/ROYCO ${ }^{\text {TMb }}$ & $4.5-225^{\mathrm{c}}$ & $\begin{array}{l}\text { Shear forces required to } \\
\text { force particle through } \\
\text { hole would disrupt } \\
\text { agglomerate. }\end{array}$ & $\begin{array}{l}\text { The hole may plug. } \\
\text { Particles may be bigger than } \\
\text { the hole. Particles are } \\
\text { assumed to be opaque. }\end{array}$ \\
\hline $\begin{array}{l}\text { Light blocking/ } \\
\text { moving laser } \\
\text { beam }\end{array}$ & $\begin{array}{l}\text { Brinkmann }{ }^{\mathrm{TM}} \\
\text { Model } 2010\end{array}$ & $0.7-150^{c}$ & & $\begin{array}{l}\text { Limited dynamic range. No } \\
\text { longer available. }\end{array}$ \\
\hline $\begin{array}{l}\text { Brownian } \\
\text { motion/Doppler } \\
\text { scattering }\end{array}$ & $\begin{array}{l}\text { Microtrac } \\
\text { Model UPA }\end{array}$ & $0.003-6.5$ & & $\begin{array}{l}\text { Very small upper bound of } \\
\text { particle size sensed. }\end{array}$ \\
\hline $\begin{array}{l}\text { Light scattering/ } \\
\text { diffraction }\end{array}$ & $\begin{array}{l}\text { Microtrac }^{{ }^{\top M}} \\
\text { Model FRA } \\
\text { Microtrac }^{{ }^{\mathrm{M}}} \\
\text { Model X-100 } \\
\text { Horiba }^{\text {TM }} \\
\text { Model LA-910 }^{\text {Model }}\end{array}$ & $\begin{array}{c}0.1-700 \\
0.04-1000 \\
0.02-1020\end{array}$ & $\begin{array}{l}\text { Large dynamic range } \\
\text { and large maximum size. } \\
\text { Horiba }{ }^{\mathrm{TM}} \text { has improved } \\
\text { stirring to suspend } \\
\text { particles. }\end{array}$ & $\begin{array}{l}\text { Proprietary calculations. } \\
\text { Surface roughness may } \\
\text { cause errors for micrometer- } \\
\text { and submicrometer-sized } \\
\text { particles. Refractive index } \\
\text { must be known (for small, } \\
\text { smooth, transparent } \\
\text { particles). }\end{array}$ \\
\hline Microscopy & $\begin{array}{l}\text { Chemical } \\
\text { (polarized light) } \\
\text { microscope or } \\
\text { scanning electron } \\
\text { microscope }\end{array}$ & & $\begin{array}{l}\text { Multipurpose equipment. } \\
\text { Can distinguish among } \\
\text { particles and observe } \\
\text { particle shapes and } \\
\text { chemical compositions. }\end{array}$ & $\begin{array}{l}\text { Preparation methods (e.g., } \\
\text { drying, gold coating) may } \\
\text { affect particle size. } \\
\text { Requires sophisticated } \\
\text { image analysis software to } \\
\text { obtain unbiased results. }\end{array}$ \\
\hline Sieving & $\begin{array}{l}\text { Standard-mesh } \\
\text { sieve sets and a } \\
\text { mechanical shaker }\end{array}$ & & $\begin{array}{l}\text { Inexpensive equipment. } \\
\text { Simple theory. }\end{array}$ & $\begin{array}{l}\text { Requires large samples and } \\
\text { a hot cell. Labor intensive. }\end{array}$ \\
\hline
\end{tabular}

a Instrument Designations, Company, Model, and Trademark Information

Brinkmann is a trademark of Brinkmann Instruments, Inc., Westbury, New York. HIAC/ROYCO is a trademark of Pacific Scientific Company, Anaheim, California. Horiba is a trademark of Horiba, Ltd., Kyoto, Japan.

Microtrac is a trademark of Leeds \& Northrup Company, North Wales, Pennsylvania.

'The HIAC/ROYCO ${ }^{\text {m }}$ Model 4300 probably was used for measuring the samples taken from Tank 241-AZ-101 in 1989.

'Measurement range in the normal configuration.

Another aspect of instrument design to consider is the sample-stirring mechanism. All of the instruments discussed herein use a stirrer to maintain the particles in suspension during the measurement. The effectiveness of this mechanism is pivotal in determining whether large, heavy particles will be detected. Most of the instruments use a stirrer (usually a magnetic stir bar with no vanes) that rotates about a vertical axis. Casting the particles up into the sensing region of the instrument depends upon a vortex action. The Horiba ${ }^{\mathrm{TM}}$ Model LA-910 has a distinctly different mechanism in that the magnetic stir bar turns about a horizontal axis. This design should propel the particles directly up into the sensing region, resulting in improved detection of large and heavy particles. 
In summary, the only instrument design attribute that obviously disqualifies any of the measurements reported in Tables 3-1, 3-2, and 3-3 is the insufficiently high upper particle size limit of the Microtrac ${ }^{\mathrm{TM}}$ Model UPA. The Horiba ${ }^{\mathrm{TM}}$ Model LA-910, on the other hand, should be quite suitable for measuring particle size because of its large upper bound and large dynamic range. The Horiba ${ }^{\mathrm{TM}}$ Model LA-910 may be subject to some bias toward smaller particle size because of the surface roughness of the particles, but there is nothing in the instrument theory or design that would indicate that the instrument could report higher particle sizes than actually exist in the sample. In fact, the special design of the stirrer in the Horiba ${ }^{\mathrm{TM}}$ Model LA-910 may be more effective in bringing larger particles into range of the sensor.

\subsubsection{Analytical Method}

Analytical methods specify how a particular instrument is applied to the measurement process. Analytical methods must accommodate special sample characteristics and how the data are to be used.

\subsubsection{Suspension of the Particulates.}

The current application of the particle size data is to the design of the WFD transport system; therefore, analyses must address the size of the particles while they are in a flowing stream. As will be discussed in Section 4.2, the particles may be agglomerated in the flowing stream. The sizes of the agglomerates affect transportability of the slurry and depends greatly on the ionic strength of the solution.

In all of the analytical methods used to obtain the data reported in Tables 3-1, 3-2, and 3-3, the particulate material must be suspended in a large amount of liquid. When selecting a suspension liquid, consideration must be given to the effect it will have on agglomeration of the particles. Dissolution of the particles also must be considered. Although the solids in HLW are primarily insoluble, soluble solids (salts) may be present as well. The soluble solids will be dissolved to a greater or lesser extent before transport, depending on the amount of dilution involved in the retrieval process.

At the 222-S Laboratory the particles most often have been measured using supernatant liquid as the suspension liquid. Using supernatant liquid for suspension maintains any agglomeration that might have occurred and prevents dissolution. When a sample of the supernatant liquid was not available elsewhere, it was sometimes centrifuged from the sample then re-combined with a small amount of the particulate material to obtain a mixture suitable for PSD. However, centrifugation may cause size classification of the particles and agglomeration. If these effects occurred, they would have been subsequently reversed by thorough re-mixing and sonication or by other means. If the level of radioactivity precluded using the natural supernatant outside of a hot cell, a simulant of the supernatant liquid may have been constructed.

At other laboratories, measurements were made under conditions where agglomerates would have been dispersed. For example, a 1:1 mixture of glycerin and water was used to suspend the particulates in a composite from Tanks 241-AZ-101 and 241-AZ-102 (see Table 3-1). This solution apparently was selected to minimize solubility of the sample, but agglomerates likely would have been dispersed because of the low ionic strength of the solution. 


\section{RPP-6427 REV 0}

Application of hydrodynamic shear forces, such as ultrasound, to the particles has been used to promote de-agglomeration. However, rapid re-agglomeration may occur if interfacial surface tension between the solid particle phase and the liquid is too high or if electrical charges carried on the particles are discharged because of the high conductivity of the solvent. As shown in Tables 3-1, 3-2, and 3-3, sonication had variable, but undramatic, results on a composite from Tanks 241-AZ-101 and 241-AZ-102 and on samples from Tanks 241-SY-102 and 241-BY-104.

Microscopy can be an effective way to detect the presence of agglomerates. However, in all the measurements cited in Tables 3-1, 3-2, and 3-3, there was only one occasion (Peters 1988) when microscopic observation was made under the same conditions as a particle size measurement. Agglomerates were detected in that measurement.

In summary, to measure the size of agglomerated particles, the suspension liquid must have appreciable ionic strength. If agglomerates are broken up by sonication, the effect is probably only temporary.

\subsubsection{Instrument Settings.}

Although the measurement process is almost completely automated using most modern particlecounting instruments, selection of instrument settings is an important aspect of the analytical method. Examples of instruments settings that may be controlled by the operator are selecting the stirring speed and entering the index of refraction of the particulate material relative to that of the liquid in the particulate.

The operator usually controls the stirring speed. The selected speed must be adequate for suspending the particles without introducing bubbles or splashing that may interfere with the measurement. On the Horiba ${ }^{\mathrm{TM}}$ Model LA-910, measurements for samples and standards are obtained at the same stirring speed to ensure that these extraneous signals are not produced.

For the Horiba ${ }^{\mathrm{TM}}$ Model LA-910 to convert the light-scattering data to a PSD, the operator must set a calculation parameter to "sharp" or "standard" to match the expected breadth of the particle distribution in the sample. This parameter apparently sets the number of iterations or error-acceptance criteria in the proprietary, iterative calculation. Setting this calculation parameter to "sharp" has produced spurious peaks for some samples; e.g., in one analysis, $85 \%$ of the particle volume was registered in the largest size bin $(890 \mu \mathrm{m}$ to $1020 \mu \mathrm{m})$. Switching the parameter to "standard" removed this anomaly completely. Acceptable central statistics (means and medians) are obtained on standard samples of monodisperse particles with both the "sharp" and "standard" settings, but the widths (standard deviations) of these narrow particle distributions are overestimated. Whereas the certificated standard distribution of the monodisperse standards are in the range of $1 \%$ to $5 \%$ relative to the mean, the results of relative standard deviations measured with the Horiba ${ }^{\mathrm{TM}}$ Model LA-910 are as high as $24 \%$ using the "standard" setting. Relative standard deviations measured with the Horiba ${ }^{\mathrm{TM}}$ Model LA-910 using the "sharp" setting are lower, usually less than $10 \%$.

The Horiba ${ }^{\mathrm{TM}}$ Model LA-910 requires that the operator enter the index of refraction of the particulate material relative to that of the liquid. However, as discussed in Section 4.1.1, this setting should have no effect for particles of the size measured in this document. 
In summary, the Horiba ${ }^{\mathrm{TM}}$ Model LA-910 instrument settings have been considered and seem to be correct.

\subsubsection{Sample Source and Selection}

The inhomogeneity of the tank wastes is well documented. When comparing the data presented in Tables 3-1, 3-2, and 3-3, it is important to consider not only the tank from which each was taken, but the position of the sample within the tank as well.

Sampling methods can also affect particle size results. Samples taken with the "grab" or "bottleon-a-string" method may favor smaller and less dense particles because the momentum of larger particles may carry them past the mouth of the bottle. Core sampling is considered the most controlled sampling method, but even core samples can be biased because of sample inhomogeneity. For instance, lumps or chunks may not enter the sampler as readily as the more fluid regions of the sample. Unfortunately, documentation of sample effects caused by sampling methods is limited, and conclusions are often based only on supposition and/or indirect evidence. For most of the data contained herein, details of sampling methods used were not available.

The sampling process does not stop when the sample gets to the laboratory. When core sample segments are removed from the sampler, the drainable liquid often is collected in a separate bottle. This liquid may contain some of the finer particulate material also. Particle size measurements generally have been made on small subsamples taken directly from the segment early in the laboratory sequence. This sample is not homogenized or blended with any other sample; therefore, the sample is not necessarily representative of the tank or even of the segment of the tank from which it was taken.

In summary, many of the particle size measurements shown in Tables 3-1, 3-2, and 3-3 may not be representative of the tank from which they were taken because of the sample inhomogeneity. However, the measurements taken with the Horiba ${ }^{\mathrm{TM}}$ Model LA-910 should be no less accurate in this respect than measurements taken with other instruments.

\subsubsection{Sample Pretreatment}

Treatment of samples from the time of sampling to the time of analysis must be examined also. Cooling unavoidably occurs for all samples taken from the tanks. Other sample pretreatment concerns include the possible drying of the samples during extended storage and the issues associated with measuring samples at different laboratories.

Ideally, particle size measurement should be taken at the temperatures expected during transport of the waste. However, all measurements have been taken at laboratory ambient temperatures because none of the instruments are equipped with temperature control. Samples are cooled from tank temperature to laboratory temperature, going through some intermediary temperature changes in the field depending on the season of the year and the time of day. For samples with soluble components, the temperature changes have dramatic effects; many samples that have been taken from tanks as liquids are entirely solid when opened in the laboratory. This problem 
is more severe for LAW samples than for HLW samples, which contain relatively less soluble material; however, it is still an issue.

Other unintended or unavoidable pretreatments include drying of the samples during extended storage. This could cause the particles to agglomerate or accrete, perhaps irreversibly. Waste samples typically are brought directly from tank farms and extruded in the 222-S Laboratory. If measurements are to be made at other laboratories, the extruded samples must be repackaged for shipment. The Horiba ${ }^{\mathrm{TM}}$ Model LA-910 measures samples at the 222-S Laboratory. Samples measured at the 222-S Laboratory usually have a simpler history of temperature and humidity compared to samples measured at other laboratories.

In summary, measurements taken with the Horiba ${ }^{\mathrm{TM}}$ Model LA-910 should not be any more adversely affected by pretreatment of the samples than measurements taken with other instruments. Because the Horiba ${ }^{\mathrm{TM}}$ Model LA-910 particle size measurements are taken on samples at the 222-S Laboratory and are not subjected to the effects of being shipped to other laboratories, the Horiba ${ }^{\mathrm{TM}}$ Model LA-910 measurements may in fact be more accurate than measurements taken with some of the other instruments.

\subsection{THE NATURE OF HIGH-LEVEL WASTE PARTICLES}

Before a bounding particle size value can be developed for use as a design basis for the WFD transfer system, more information is needed about the nature of sludge particles. This section addresses the nature of the particles in the HLW slurries.

Metallic hydroxides and hydrated oxide particulates can be expected to agglomerate in solutions of moderate ionic strength such as are present in HLW. Agglomeration is caused by neutralization of the surface charge on the particles. Surface charge is required to keep the particles dispersed, but the presence of electrolytes in the solution causes these charges to be neutralized (Sennett and Olivier 1965).

During retrieval, the waste is subjected to sluicing and/or passed through a mixer pump several times, then passed through a multistage turbine transfer pump. This action may break up agglomerated particles. However, because the waste usually is not diluted extensively, the ionic strength will still be substantial and the particles would tend to re-agglomerate when mixing ceases. These statements are supported by theory and have been demonstrated for boehmite and ferric hydroxide particles (PNL-10761). When the structures of particles in HLW from Tanks 241-AY-101 and 241-SY-102 were examined microscopically, agglomerates were observed (Peters 1988; PNNL-11352). However, these agglomerated waste particles may disperse into their component particles when placed into liquids of low ionic strength.

Agglomeration will affect settling rate and slurry transport. It is useful to consider Stokes Law to gain a qualitative understanding of the effect of agglomeration. Stokes Law shows that the terminal velocity of a spherical particle falling freely in a quiescent liquid is proportional to its cross sectional area and the difference between density of the particle and the density of the liquid. Because agglomerates contain a good deal of interstitial liquid, they are expected to be less dense than entirely solid particles. Consequently, agglomerates might be expected to settle 


\section{RPP-6427 REV 0}

more slowly than solid particles. For instance, an agglomerate particle in which half the volume was interstitial liquid would settle at half the velocity of a particle of the same size that is entirely solid. However, most agglomerates contain hundreds or thousands of individual particles. Thus, their huge size usually far outweighs the smaller density, and the particles settle considerably faster. In fact, this is the theory behind the many industrial flocculation/clarification processes.

Microscopic observation of wastes can provide some clues about the densities of agglomerates. For instance, the transmission electron microscope pictures of waste particles from Tank 241-SY-102 (PNNL-11352) and Tank 241-AW-105 (PNL-10761) show accretions containing large numbers of particles in which perhaps half the volume is liquid. However, methods of preparing the sample for microscopic examination must be considered carefully. For instance, in PNNL-11352, drying of the sample left much solid sodium hydroxide in the residual solid. Sodium hydroxide usually is dissolved completely in tank wastes.

Despite indications from laboratory studies that the particles are agglomerated, little is known about how readily the particles may be broken up by pump action or by turbulence caused by flow in a pipe. The few studies that have been done indicate that the tendency toward agglomeration is strong, very severe mechanical treatment is required to break the agglomerates, and the agglomerates will re-form when the mechanical treatment is halted. The particle sizes in a simulant of the waste in Tank 241-C-103 were observed as the material was recirculated through a particle size instrument (PNL-10761). After $2 \mathrm{~h}$, about half of the material had been broken into particles of approximately $1 \mu \mathrm{m}$ in size; however, after $6 \mathrm{~h}$ this process was still incomplete.

Sonication disrupted the agglomerates in Tank 241-SY-102 waste only slightly (PNNL-11352). Sonication also produced only slight reduction in the size of particles from Tank 241-BY-104 (PNNL-11278). In a sample from Tank 241-S-111, sonication appeared to actually increase the particle size (PNNL-1 1636). Much data was obtained during the recent test of the mixer pumps in Tank 241-AZ-101 (RPP-6548). Thorough analysis of these data likely will provide additional information about the effects of pumps on particle size and settling rates.

It is often assumed that HLW materials have a very fine particle size because they have a smooth and clay-like consistency when moist. (Hence, the term "sludge" commonly used to describe HLW materials.) On the other hand, if the material contained particles exceeding $100 \mu \mathrm{m}$ in diameter, such as have been measured, one might think the material would be gritty. After all, the abrasive particles in 150-grit sandpaper have diameters of approximately $100 \mu \mathrm{m}$. However, the presence of fragile agglomerate particles that can flex or break with only slight mechanical force would permit the "sludgy" texture to be reconciled with the large particle sizes measured.

\subsection{STATISTICAL ANALYSIS OF PARTICLE SIZE DATA FOR HIGH-LEVEL WASTE}

This section presents statistical calculations used to define a bounding value for the size of particles in the waste to be delivered to the Waste Treatment and Immobilization Plant. The results of this statistical analysis provide information required for the slurry flow model calculations. The slurry flow calculations require a "bounding" median particle diameter size and a "typical" PSD. The median particle size and distribution are needed to calculate the 


\section{RPP-6427 REV 0}

parameter "p" for the Rosin-Rammler distribution (RPP-5346, p. F-19). Estimates of the median particle size diameter and a "typical" PSD are reported in this section.

The statistical calculations in this section are based on PSD data obtained with the Horiba ${ }^{\mathrm{TM}}$ Model LA-910 on HLW samples. Measurements of LAW samples were not considered in this analysis because the solids in LAW must be dissolved before delivery. Wastes to be delivered to the Waste Treatment and Immobilization Plant in Phase 2 were not used in this analysis because measurements of those wastes also include much solid material that will be dissolved before being transported by pipe. However, the particle sizes of insoluble solids in Phase 2 wastes should not be greatly different from those in Phase 1 wastes.

The statistical analysis makes use of data obtained with the Horiba ${ }^{\mathrm{TM}}$ Model LA-910 only. This choice was made to ensure conservatism in the calculations; as mentioned previously, the particle size results determined using the Horiba ${ }^{\mathrm{TM}}$ Model LA-910 generally are larger than the results obtained using other particle size instruments. The reasons for this difference are unknown. A possible reason is that agglomeration was more prevalent in the $\mathrm{Horiba}^{\mathrm{TM}}$ Model LA-910 measurements because the ionic strength of the suspending liquid was higher. Another reason may be related to the stirring capabilities of the Horiba ${ }^{\mathrm{TM}}$ Model LA-910. The improved stirring may have permitted the instrument to register particles that other instruments have missed.

The PSD data obtained with the Horiba ${ }^{\mathrm{TM}}$ Model LA-910 are available for three HLW tanksTanks 241-AW-103, 241-AZ-101, and 241-C-104. If these three tanks are assumed to be a random sample of the whole population of ten HLW tanks, then the statistical results (e.g., means and medians) are unbiased estimates of the corresponding values for the population. To assess the soundness of this assumption, information about the sources of the waste stored in the ten HLW tanks (HNF-2177) was evaluated. This information is presented in Table 4-2. The rows containing data obtained using the Horiba ${ }^{\mathrm{TM}}$ Model LA-910 are shaded. As shown in Table 4-2, the solid material in the three tanks selected for sampling represents approximately $40 \%$ of the solid material in all ten of the HLW tanks. Information about the volumes of waste from the various sources is incomplete. However, discounting salt cake and double-shell slurry feed (which should be dissolved before transport), the tanks appear to contain wastes mostly from the Plutonium-Uranium Extraction (PUREX) facility.

The only distinctly different and insoluble HLW type that is present in appreciable quantities and has not been analyzed by the Horiba ${ }^{\mathrm{TM}}$ Model LA-910 is the bismuth phosphate waste in Tank 241-C-107. This waste represents only $12 \%$ of the solid waste volume in the HLW tanks, but greater than $80 \%$ of the volume in Tank $241-\mathrm{C}-107$. Therefore, it was concluded that the sampled wastes should be representative of the HLW tanks, with the possible exception of Tank 241-C-107.

There were a total of 21 PSDs obtained with the Horiba ${ }^{\mathrm{TM}}$ Model LA-910 for the $3 \mathrm{HLW}$ tanks: 2 samples from Tank 241-AZ-101, 15 samples from Tank 241-AW-103, and 4 samples from Tank 241-C-104. The data were reported in RPP-5798, O'Rourke 2000a, and O'Rourke 2000b; electronic copies of the distribution data were obtained from the Tank Characterization Database (DOE 2000). Because the numbers of samples taken from each tank are different, the 


\section{RPP-6427 REV 0}

Table 4-2. Major Waste Types in the High-Level Waste Tanks. ${ }^{\mathrm{a}}$

\begin{tabular}{|c|c|c|c|c|c|c|}
\hline \multirow{3}{*}{ Tank } & \multicolumn{3}{|c|}{ Phases } & \multicolumn{3}{|l|}{ Waste Source (Both Phases) } \\
\hline & \multirow{2}{*}{ Type } & \multicolumn{2}{|c|}{ Volume } & \multirow{2}{*}{ Type } & \multicolumn{2}{|c|}{ Volume } \\
\hline & & $\mathbf{m}^{3}$ & (kgal) & & $\mathbf{m}^{3}$ & (kgal) \\
\hline W & Wor & (3) & 196. & 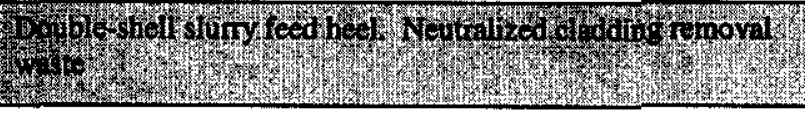 & 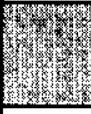 & Why \\
\hline \multirow[t]{2}{*}{ 241-AW-104 } & Liquid & 3,138 & (829) & A saltcake & 712 & $(188)$ \\
\hline & Solid & 1,098 & $(290)$ & Zirconium cladding waste & 19 & (5) \\
\hline \multirow[t]{5}{*}{ 241-AY-101 } & \multirow[t]{3}{*}{ Liquid } & \multirow[t]{3}{*}{129} & \multirow[t]{3}{*}{ (34) } & B Plant waste & 68 & (18) \\
\hline & & & & Cesium recovery waste & 27 & $(7)$ \\
\hline & & & & PUREX sludge & 30 & (8) \\
\hline & \multirow[t]{2}{*}{ Solid } & \multirow[t]{2}{*}{408} & \multirow[t]{2}{*}{$(108)$} & Precipitate from evaporator slurry & 163 & (43) \\
\hline & & & & Unknown sources & 121 & (32) \\
\hline \multirow[t]{2}{*}{$241-A Y-102$} & Liquid & 3,017 & (797) & \multirow{2}{*}{$\begin{array}{l}\text { Evaporator slurry, double-shell slurry feed from } \\
242-A \text { Evaporator; vitrification process test wastes; dilute } \\
\text { noncomplexed waste from B Plant, T Plant, and the } 100,300 \text {, } \\
\text { and } 400 \text { Areas; HLW from strontium purification at B Plant; } \\
\text { filtrate from Waste Encapsulation and Storage Facility }\end{array}$} & & \\
\hline & Solid & 83 & $(22)$ & & & \\
\hline W. & 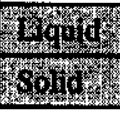 & 36x & (1) & 3.7. & 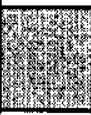 & 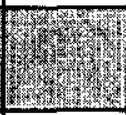 \\
\hline \multirow[t]{2}{*}{ 241-AZ-102 } & Liquid & 2,854 & $(754)$ & \multirow[t]{2}{*}{ PUREX HLW and perhaps PUREX LLW and B Plant LLW } & & \\
\hline & Solid & 394 & (104) & & & \\
\hline 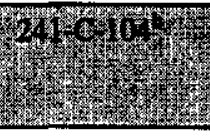 & (x) & 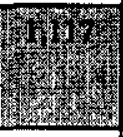 & W.t. & 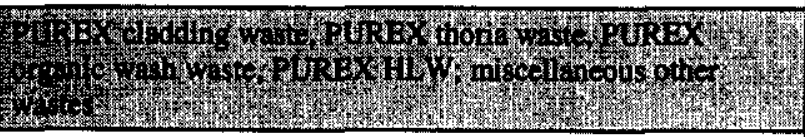 & 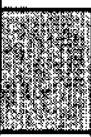 & 2.t. \\
\hline \multirow[t]{4}{*}{$241-C-106$} & \multirow[t]{2}{*}{ Liquid } & \multirow[t]{2}{*}{121} & \multirow[t]{2}{*}{$(32)$} & Uranium recovery waste & 57 & $(15)$ \\
\hline & & & & PUREX cladding waste & 129 & (34) \\
\hline & \multirow[t]{2}{*}{ Solid } & \multirow[t]{2}{*}{746} & \multirow[t]{2}{*}{ (197) } & AR Vault waste & 363 & (96) \\
\hline & & & & B Plant LLW & 197 & $(52)$ \\
\hline \multirow[t]{3}{*}{ 241-C-107 } & \multirow[t]{3}{*}{ Solid } & \multirow[t]{3}{*}{973} & \multirow[t]{3}{*}{$(257)$} & $\mathrm{BiPO}_{4}$ first-cycle sludge & 799 & (211) \\
\hline & & & & PUREX cladding waste & 133 & $(35)$ \\
\hline & & & & Hot semi-works and strontium-recovery waste & 42 & (11) \\
\hline \multirow[t]{2}{*}{$241-S Y-102$} & Liquid & 2,438 & $(644)$ & \multirow{2}{*}{$\begin{array}{l}\text { 242-S Evaporator saltcake, Z Plant waste, T Plant } \\
\text { decontamination waste }\end{array}$} & & \\
\hline & Solid & 333 & $(88)$ & & & \\
\hline TOTAL & Liquid & 15,410 & $(4,071)$ & & & \\
\hline & Solid & 6,705 & $(1,771)$ & & & \\
\hline
\end{tabular}

HLW = high-level waste.

LLW $=$ low-level waste.

PUREX= Plutonium Uranium Extraction (facility).

"Data taken from HNF-2177, 1998, Tank-by-Tank Safety Status Evaluation, Rev. 0-B, Lockheed Martin Hanford Corporation, Richland, Washington.

bShading indicates that data for this tank was obtained using the Horiba ${ }^{\mathrm{TM}}$ Model LA-910. (Horiba is a trademark of Horiba, Ltd., Kyoto, Japan.) 


\section{RPP-6427 REV 0}

data are unbalanced. The lack of balance restricts the statistical methods that can be used to analyze the data. A Process Control Engineer reviewed the statistical treatment of the data. Documentation of the review is provided in Appendix A.

The 21 sets of PSD data are reported in Table 4-3. The first and last columns report the upper limits of the particle size bins in micrometers $(\mu \mathrm{m})$. The numbers in the body of the table are the percent of the total particulate volume in each bin. For each PSD the maximum percent in a bin is shaded. The Horiba ${ }^{\mathrm{TM}}$ Model LA-910 does not report values greater than $1020 \mu \mathrm{m}$. Consequently, the PSDs are conditional distributions; the PSDs are conditional on the particle diameters being less than $1020 \mu \mathrm{m}$. The columns in Table 4-3 contain very similar data. Therefore, the 21 PSDs are not very different between samples within tanks or between tanks. This is some evidence that the 3 tanks are from the same population and that the data therefore can be pooled. However, all of the samples were prepared for particle analysis by a common method. Consequently, the PSDs may be similar because of common sample preparation (i.e., the sample preparation may bias the PSDs).

There are three statistical analysis methods for analyzing the PSD data: (1) an analysis of the mean percentile points, (2) an analysis of variance (ANOVA), and (3) an analysis of the tank cumulative means. The statistical results are in the form of means, the one-sided $95 / 95$ tolerance limits, and the upper limits to one-sided $95 \%$ confidence limits. The $95 / 95$ tolerance limits and upper limits to $95 \%$ confidence limits are defined as follows.

A $95 / 95$ tolerance limit is a $95 \%$ confidence limit on $95 \%$ of the population. The upper limit to a one-sided $95 / 95$ tolerance limit is

$$
\text { 95/95TL }=\text { mean }+7.655 \times \text { S.D. }
$$

where "mean" is an estimate of the population mean, S.D. is the "standard deviation," and 7.655 is the tolerance factor for the normal distribution based on three observations (three means). The tolerance factors are tabulated in Table A7 of the National Bureau of Standards Handbook 91 (1963). The interpretation associated with a tolerance limit is "we are $95 \%$ confident that at least $95 \%$ of the population will have a value less than the computed number 95/95TL."

The upper limit to a one-sided $95 \%$ confidence interval on a mean is

$$
\mathrm{UL}(95 \%)=\text { mean }+2.920 \times \text { S.D. }(\text { mean })
$$

where "mean" is an estimate of the population mean and S.D.(mean) is the "standard deviation of the mean." Usually the standard deviation of the mean is the ordinary standard deviation divided by the square root of the number of observations. The number 2.920 is the quantile from Student's $t$ distribution for a one-sided $95 \%$ confidence interval with two degrees of freedom. The interpretation associated with a one-sided confidence limit is "we are $95 \%$ confident that the population mean will have a value less than the computed number UL(95\%)." 
Table 4-3. Particle Volume Distribution Data by Tank and Sample, the Maximum in Each is Shaded (units are given in percentage).

\begin{tabular}{|c|c|c|c|c|c|c|c|c|c|c|c|c|c|c|c|c|c|c|c|c|c|c|}
\hline 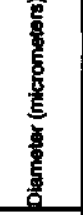 & 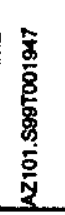 & $\begin{array}{l}\frac{9}{3} \\
\frac{8}{8} \\
\frac{5}{8} \\
\frac{8}{8} \\
\frac{5}{2}\end{array}$ & 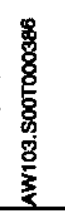 & $\begin{array}{l}\mathbf{3} \\
8 \\
8 \\
8 \\
8 \\
8 \\
8 \\
\frac{8}{5} \\
3\end{array}$ & $\begin{array}{l}\mathbf{8} \\
8 \\
8 \\
8 \\
8 \\
8 \\
8 \\
8 \\
\frac{8}{5} \\
\end{array}$ & $\begin{array}{l}8 \\
8 \\
8 \\
8 \\
8 \\
8 \\
8 \\
8 \\
\frac{5}{3} \\
\end{array}$ & $\begin{array}{l}8 \\
8 \\
8 \\
8 \\
8 \\
8 \\
8 \\
8 \\
\frac{8}{3} \\
\end{array}$ & $\begin{array}{l}\overline{8} \\
8 \\
8 \\
8 \\
8 \\
8 \\
8 \\
8 \\
\frac{8}{3} \\
\end{array}$ & $\begin{array}{l}8 \\
8 \\
8 \\
8 \\
8 \\
8 \\
8 \\
8 \\
8 \\
3 \\
\end{array}$ & 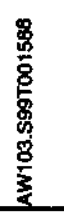 & 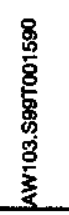 & 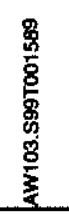 & 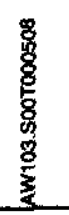 & 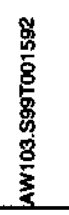 & 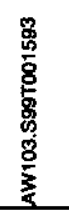 & $\begin{array}{l}\text { 点 } \\
\frac{5}{8} \\
8 \\
8 \\
8 \\
8 \\
8 \\
8\end{array}$ & 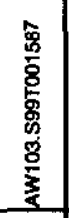 & $\frac{\stackrel{1}{\delta}}{\frac{\delta}{0}}$ & $\begin{array}{l}\frac{8}{8} \\
\frac{8}{3} \\
\end{array}$ & 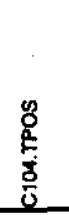 & $\frac{5}{\frac{5}{5}}$ & 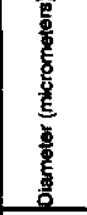 \\
\hline $\begin{array}{c}0.17 \\
0.20 \\
0.23 \\
\ldots \\
5.12 \\
5.87 \\
6.72 \\
7.70 \\
8.82 \\
10.10 \\
11.56 \\
13.25 \\
15.17 \\
17.38 \\
19.90 \\
22.80 \\
26.11 \\
29.91 \\
34.25 \\
39.23 \\
14.94 \\
51.47 \\
58.96 \\
67.52 \\
77.34 \\
68.58 \\
101.46 \\
116.21 \\
133.10 \\
152.45 \\
174.62 \\
200.00 \\
228.09 \\
262.38 \\
300.52 \\
344.21 \\
394.24 \\
451.56 \\
517.20 \\
592.39 \\
678.50 \\
777.14 \\
890.12 \\
1019.51\end{array}$ & $\begin{array}{l}0.11 \\
0.23 \\
0.41 \\
0.77 \\
1.06 \\
1.23 \\
1.37 \\
1.37 \\
1.48 \\
1.54 \\
1.62 \\
1.91 \\
2.26 \\
2.76 \\
3.50 \\
4.49 \\
5.63 \\
6.90 \\
8.05 \\
8.10 \\
1.20 \\
8.22 \\
7.58 \\
6.49 \\
5.08 \\
3.46 \\
2.06 \\
1.08 \\
0.56 \\
0.31 \\
0.17\end{array}$ & $\begin{array}{l}0.16 \\
0.33 \\
0.52 \\
0.73 \\
0.98 \\
1.13 \\
1.12 \\
1.06 \\
1.16 \\
1.36 \\
1.48 \\
1.68 \\
1.96 \\
2.37 \\
2.90 \\
3.61 \\
4.51 \\
5.69 \\
7.14 \\
8.99 \\
10.77 \\
11.79 \\
9.38 \\
5.08 \\
1.37 \\
0.19 \\
0.11\end{array}$ & $\begin{array}{l}0.60 \\
2.74 \\
2.26 \\
2.12 \\
2.49 \\
3.29 \\
3.70 \\
2.57 \\
2.54 \\
2.70 \\
2.53 \\
3.79 \\
7.40 \\
20.20 \\
9.19 \\
1.12\end{array}$ & $\begin{array}{c}0.17 \\
0.50 \\
0.62 \\
0.43 \\
0.39 \\
0.50 \\
0.77 \\
0.86 \\
1.25 \\
1.29 \\
1.79 \\
1.61 \\
2.09 \\
1.90 \\
2.09 \\
2.83 \\
4.03 \\
3.88 \\
4.11 \\
4.07 \\
7.22 \\
13.74 \\
20.4 \\
1.12 \\
1.12\end{array}$ & $\begin{array}{c}0.13 \\
0.28 \\
0.52 \\
0.95 \\
0.90 \\
1.87 \\
1.79 \\
2.40 \\
1.36 \\
1.69 \\
1.32 \\
1.57 \\
2.78 \\
4.47 \\
4.48 \\
4.48 \\
3.94 \\
6.61 \\
12.42 \\
21.15 \\
0.14 \\
1.74\end{array}$ & $\begin{array}{l}0.23 \\
2.37 \\
2.65 \\
2.32 \\
3.37 \\
4.21 \\
5.01 \\
2.77 \\
2.45 \\
2.68 \\
3.11 \\
5.89 \\
12.85 \\
21.04 \\
3.22 \\
0.19\end{array}$ & $\begin{array}{c}0.28 \\
0.46 \\
0.47 \\
0.39 \\
0.33 \\
0.47 \\
0.77 \\
1.05 \\
1.52 \\
1.45 \\
1.61 \\
1.56 \\
2.00 \\
1.86 \\
2.19 \\
2.91 \\
3.96 \\
4.07 \\
4.06 \\
4.24 \\
7.50 \\
14.50 \\
.40 \\
20.14 \\
1.01\end{array}$ & $\begin{array}{c}0.21 \\
0.38 \\
0.39 \\
0.27 \\
0.25 \\
0.35 \\
0.61 \\
0.72 \\
1.20 \\
1.44 \\
1.76 \\
1.52 \\
1.89 \\
1.04 \\
1.33 \\
1.62 \\
3.86 \\
4.90 \\
5.72 \\
3.47 \\
4.36 \\
5.42 \\
14.12 \\
10.00 \\
13.86\end{array}$ & $\begin{array}{c}0.27 \\
0.85 \\
1.77 \\
1.60 \\
2.57 \\
1.70 \\
1.68 \\
2.67 \\
4.52 \\
7.15 \\
6.37 \\
6.05 \\
5.45 \\
5.14 \\
7.52 \\
12.96 \\
\vdots 11.86 \\
2.25 \\
0.18\end{array}$ & $\begin{array}{l}0.10 \\
1.57 \\
2.62 \\
2.68 \\
1.13 \\
1.18 \\
0.98 \\
0.91 \\
1.87 \\
3.30 \\
4.04 \\
4.92 \\
4.81 \\
5.48 \\
6.35 \\
8.89 \\
14.45 \\
15.47 \\
.4 . \\
3.73\end{array}$ & $\begin{array}{c}1.26 \\
2.94 \\
3.21 \\
0.85 \\
0.86 \\
0.62 \\
0.66 \\
1.80 \\
3.60 \\
4.28 \\
5.15 \\
4.06 \\
4.41 \\
5.19 \\
9.11 \\
14.30 \\
14.96 \\
17.75 \\
0.26\end{array}$ & $\begin{array}{c}1.60 \\
5.64 \\
2.56 \\
1.13 \\
1.57 \\
3.81 \\
7.17 \\
9.74 \\
12.08 \\
9.62 \\
6.21 \\
6.12 \\
7.29 \\
8.60 \\
8.10 \\
1.66 \\
0.40\end{array}$ & $\begin{array}{c}0.54 \\
1.22 \\
1.96 \\
0.81 \\
0.46 \\
0.74 \\
1.50 \\
2.76 \\
3.51 \\
4.54 \\
4.93 \\
4.68 \\
5.77 \\
8.18 \\
11.42 \\
11.40 \\
14.00 \\
11.33 \\
6.69 \\
0.87\end{array}$ & $\begin{array}{c}0.16 \\
1.97 \\
2.23 \\
1.73 \\
0.75 \\
0.88 \\
0.72 \\
0.78 \\
1.64 \\
2.83 \\
3.20 \\
3.83 \\
3.76 \\
4.98 \\
6.21 \\
8.26 \\
0.3 .4 \\
5.77 \\
11.38 \\
15.44 \\
13.41\end{array}$ & $\begin{array}{c}1.38 \\
2.42 \\
0.58 \\
0.11 \\
0.21 \\
0.88 \\
2.70 \\
7.66 \\
16.86 \\
\\
1.1 .77 \\
7.07 \\
5.04 \\
5.30 \\
6.44 \\
5.98 \\
5.04 \\
1.42\end{array}$ & $\begin{array}{c}0.77 \\
3.27 \\
2.14 \\
1.25 \\
2.05 \\
3.70 \\
6.43 \\
6.02 \\
7.06 \\
8.37 \\
9.97 \\
16.18 \\
10.88 \\
1.31\end{array}$ & $\begin{array}{c}1.38 \\
5.37 \\
2.64 \\
1.08 \\
1.20 \\
2.94 \\
5.33 \\
9.16 \\
16.88 \\
12.50 \\
0.22 \\
4.71 \\
3.00 \\
1.96 \\
1.54 \\
1.00 \\
1.41 \\
0.99\end{array}$ & $\begin{array}{l}0.15 \\
0.21 \\
0.27 \\
0.33 \\
0.37 \\
0.41 \\
0.44 \\
0.46 \\
0.44 \\
0.45 \\
0.44 \\
0.44 \\
0.59 \\
0.76 \\
0.94 \\
1.14 \\
1.35 \\
1.62 \\
1.94 \\
2.33 \\
2.81 \\
3.34 \\
3.95 \\
4.72 \\
5.34 \\
5.93 \\
7.56 \\
9.35 \\
12.28 \\
70.014 \\
4.72 \\
1.57 \\
0.42 \\
0.23 \\
0.13\end{array}$ & $\begin{array}{l}0.30 \\
0.76 \\
0.85 \\
0.54 \\
0.45 \\
0.56 \\
0.77 \\
0.74 \\
0.65 \\
0.49 \\
0.56 \\
1.09 \\
1.63 \\
2.40 \\
2.17 \\
2.29 \\
2.99 \\
3.37 \\
4.01 \\
4.49 \\
5.10 \\
6.27 \\
10.89 \\
15.37 \\
14.63 \\
0.46 \\
0.36\end{array}$ & $\begin{array}{c}0.47 \\
0.81 \\
0.70 \\
0.39 \\
0.33 \\
0.44 \\
0.68 \\
0.23 \\
0.81 \\
0.68 \\
0.81 \\
1.23 \\
1.69 \\
2.21 \\
2.02 \\
2.31 \\
3.15 \\
3.60 \\
4.21 \\
4.61 \\
5.29 \\
6.65 \\
11.53 \\
14.91 \\
13.03 \\
0.23\end{array}$ & $\begin{array}{l}1.36 \\
1.04 \\
0.64 \\
0.37 \\
0.22 \\
0.25 \\
0.44 \\
0.80 \\
1.65 \\
1.90 \\
1.98 \\
2.30 \\
2.80 \\
3.76 \\
4.63 \\
4.70 \\
5.05 \\
5.58 \\
5.62 \\
6.01 \\
6.86 \\
7.96 \\
8.79 \\
1.62 \\
1.62 \\
5.06 \\
1.89\end{array}$ & $\begin{array}{c}0.17 \\
0.20 \\
0.23 \\
\ldots \\
5.12 \\
5.87 \\
6.72 \\
7.70 \\
8.82 \\
10.10 \\
11.56 \\
13.25 \\
15.17 \\
17.39 \\
19.90 \\
22.80 \\
26.11 \\
29.91 \\
34.25 \\
39.23 \\
44.94 \\
51.47 \\
58.95 \\
67.52 \\
77.34 \\
88.58 \\
101.46 \\
116.21 \\
133.10 \\
152.45 \\
174.62 \\
200.00 \\
229.08 \\
262.38 \\
300.52 \\
344.21 \\
394.24 \\
451.56 \\
517.20 \\
592.39 \\
678.50 \\
77.14 \\
890.12 \\
1019.51\end{array}$ \\
\hline
\end{tabular}

\subsubsection{Analysis of Mean Percentile Points}

Table 4-4 lists the mean percent of the particle volume distribution in each of the bins for each of the three tanks measured using the Horiba ${ }^{\mathrm{TM}}$ Model LA-910. The first column reports the upper limits of the particle size bins in micrometers $(\mu \mathrm{m})$. The numbers in the body of the table are the mean percent of the particulate volume distribution (i.e., the ordinary arithmetic mean of the numbers) given by tank. The column Grand.Mean is the arithmetic mean of the three tank means. Figure 4-1 consists of graphs of the means given in Table 4-4. The first three graphs are the three tank means. The fourth graph is a composite of the three tank means and the Grand.Mean. It is evident that the particle distributions given by the three means and the Grand.Mean are not very different from each other. 
Table 4-4. Mean Volume Percent in Bins (units are given in percentage).

\begin{tabular}{|c|c|c|c|c|}
\hline 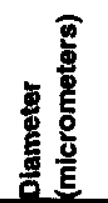 & $\begin{array}{l}\frac{c}{9} \\
\frac{8}{5} \\
\frac{5}{5} \\
\frac{0}{y}\end{array}$ & $\begin{array}{l}5 \\
\frac{9}{9} \\
5 \\
8 \\
\end{array}$ & $\begin{array}{l}\frac{5}{8} \\
\frac{5}{6} \\
\frac{0}{0}\end{array}$ & 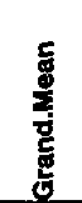 \\
\hline 0.20 & 0.00 & 0.05 & 0.00 & 0.02 \\
\hline 4.47 & 0.00 & 0.00 & 0.15 & 0.05 \\
\hline 5.12 & 0.00 & 0.00 & 0.38 & 0.13 \\
\hline 5.87 & 0.00 & 0.04 & 0.50 & 0.18 \\
\hline 6.72 & 0.00 & 0.09 & 0.62 & 0.24 \\
\hline 7.70 & 0.00 & 0.10 & 0.56 & 0.22 \\
\hline 8.82 & 0.09 & 0.08 & 0.38 & 0.18 \\
\hline 10.10 & 0.22 & 0.10 & 0.36 & 0.23 \\
\hline 11.56 & 0.37 & 0.18 & 0.47 & 0.34 \\
\hline 13.25 & 0.57 & 0.36 & 0.68 & 0.54 \\
\hline 15.17 & 0.88 & 0.84 & 0.91 & 0.88 \\
\hline 17.38 & 1.09 & 2.46 & 0.95 & 1.50 \\
\hline 19.90 & 1.18 & 1.93 & 0.89 & 1.33 \\
\hline 22.80 & 1.22 & 1.69 & 1.03 & 1.31 \\
\hline 26.11 & 1.27 & 1.54 & 1.43 & 1.41 \\
\hline 29.91 & 1.42 & 2.36 & 1.96 & 1.91 \\
\hline 34.25 & 1.51 & 3.25 & 2.54 & 2.43 \\
\hline 39.23 & 1.64 & 3.82 & 2.51 & 2.66 \\
\hline 44.94 & 1.94 & 5.61 & 2.75 & 3.43 \\
\hline 51.47 & 2.32 & 6.52 & 3.34 & 4.06 \\
\hline 58.95 & 2.84 & 5.66 & 3.63 & 4.04 \\
\hline 67.52 & 3.56 & 6.19 & 4.14 & 4.63 \\
\hline 77.34 & 4.50 & 7.16 & 4.69 & 5.45 \\
\hline 88.58 & 5.66 & 9.61 & 5.42 & 6.90 \\
\hline 101.46 & 7.02 & 10.67 & 6.42 & 8.03 \\
\hline 116.21 & 8.51 & 9.71 & 9.29 & 9.17 \\
\hline 133.10 & 9.93 & 9.51 & 11.24 & 10.23 \\
\hline 152.45 & 10.48 & 4.26 & 10.13 & 8.29 \\
\hline 174.62 & 10.32 & 4.02 & 9.67 & 8.00 \\
\hline 200.00 & 8.47 & 1.30 & 2.48 & 4.08 \\
\hline 229.08 & 5.78 & 0.89 & 3.07 & 3.25 \\
\hline 262.38 & 3.22 & 0.00 & 3.14 & 2.12 \\
\hline 300.52 & 1.83 & 0.00 & 2.50 & 1.44 \\
\hline 344.21 & 1.08 & 0.00 & 1.18 & 0.75 \\
\hline 394.24 & 0.54 & 0.00 & 0.39 & 0.31 \\
\hline 451.56 & 0.28 & 0.00 & 0.11 & 0.13 \\
\hline 517,20 & 0.15 & 0.00 & 0.06 & 0.07 \\
\hline 592.39 & 0.09 & 0.00 & 0.03 & 0.04 \\
\hline
\end{tabular}




\section{RPP-6427 REV 0}
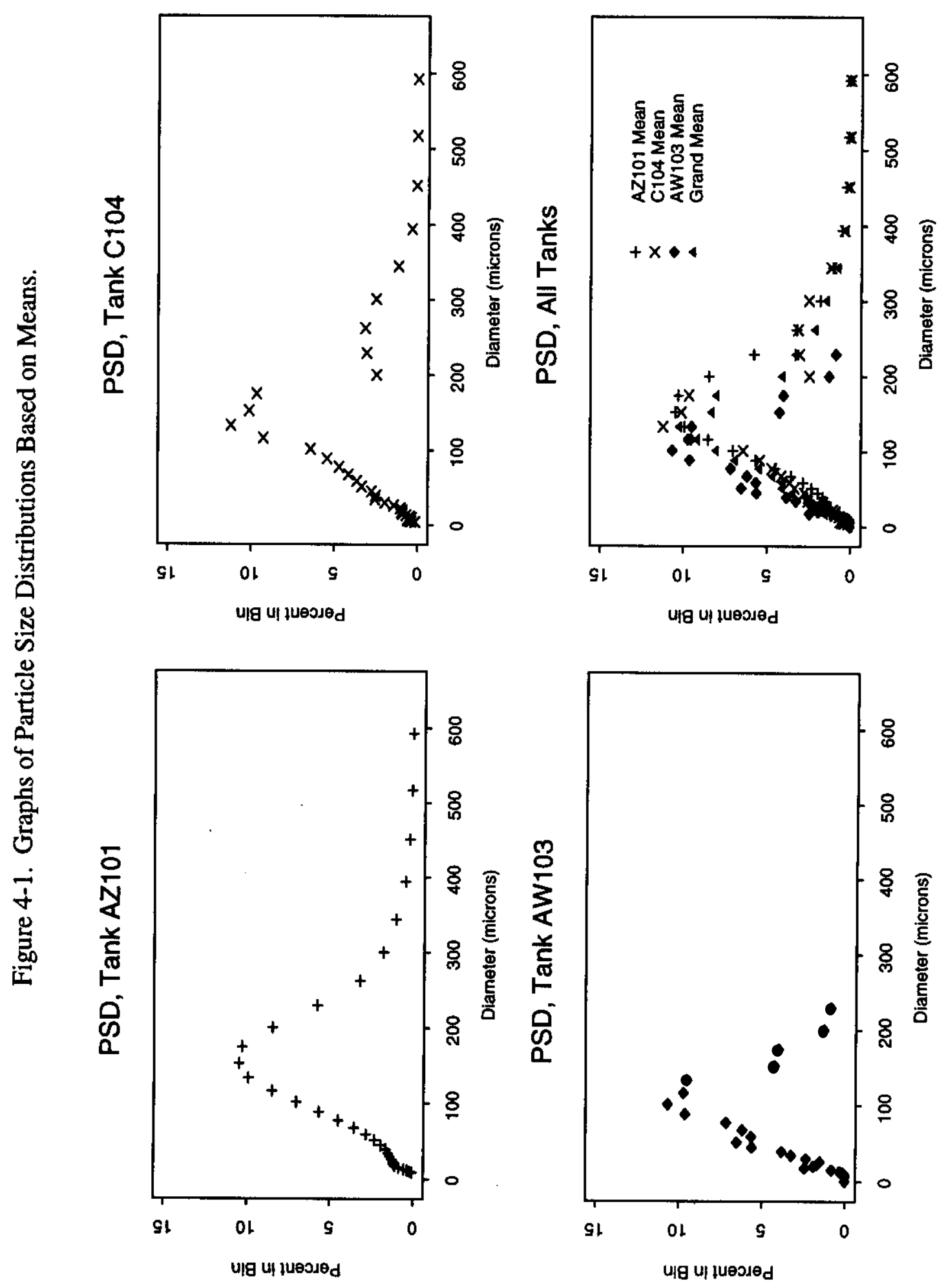
Table 4-5 lists the cumulative particle volume percent in the bins (i.e., the cumulative sum of the elements in the columns in Table 4-3). The first and last columns report the upper limits of the particle size bins in micrometers $(\mu \mathrm{m})$. The numbers in the body of the table are the percent of the cumulative particulate volume in each bin.

Table 4-5. Cumulative Particle Volume Distribution (units are given in percentage).

\begin{tabular}{|c|c|c|c|c|c|c|c|c|c|c|c|c|c|c|c|c|c|c|c|c|c|c|}
\hline $\begin{array}{l}\overline{\mathbf{5}} \\
\mathbf{5} \\
\mathbf{5} \\
\mathbf{5} \\
\mathbf{5} \\
\mathbf{5} \\
\mathbf{5} \\
\end{array}$ & $\begin{array}{l}\mathbf{5} \\
\frac{5}{8} \\
\frac{5}{8} \\
5 \\
\frac{5}{2}\end{array}$ & $\begin{array}{l}\frac{0}{0} \\
\frac{5}{8} \\
\frac{8}{8} \\
\frac{5}{0} \\
\frac{5}{2} \\
2\end{array}$ & $\begin{array}{l}8 \\
8 \\
8 \\
8 \\
8 \\
8 \\
8 \\
8 \\
8\end{array}$ & 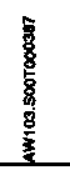 & $\begin{array}{l}8 \\
8 \\
8 \\
8 \\
8 \\
8 \\
8 \\
5 \\
\end{array}$ & $\begin{array}{l}8 \\
8 \\
8 \\
8 \\
8 \\
8 \\
8 \\
8 \\
3\end{array}$ & $\begin{array}{l}8 \\
8 \\
8 \\
8 \\
8 \\
8 \\
8 \\
8 \\
\\
\end{array}$ & $\begin{array}{l}\overline{8} \\
8 \\
8 \\
8 \\
8 \\
8 \\
8 \\
\end{array}$ & $\begin{array}{l}\mathbf{8} \\
8 \\
6 \\
8 \\
8 \\
8 \\
8 \\
2\end{array}$ & $\begin{array}{l}\frac{8}{5} \\
\frac{8}{8} \\
8 \\
8 \\
8 \\
8 \\
\frac{8}{5}\end{array}$ & $\begin{array}{l}8 \\
\frac{8}{5} \\
8 \\
8 \\
8 \\
8 \\
8 \\
8 \\
\end{array}$ & $\begin{array}{l}\frac{8}{8} \\
\frac{8}{8} \\
\frac{8}{8} \\
\frac{8}{8} \\
\frac{3}{3}\end{array}$ & $\begin{array}{l}8 \\
8 \\
8 \\
8 \\
8 \\
8 \\
8 \\
\frac{5}{3} \\
\end{array}$ & 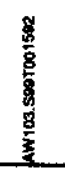 & 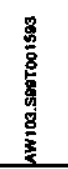 & 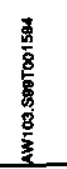 & $\begin{array}{l}5 \\
\frac{5}{8} \\
8 \\
8 \\
8 \\
8 \\
8 \\
\end{array}$ & $\frac{5}{6}$ & $\begin{array}{l}0 \\
8 \\
\vdots \\
0\end{array}$ & $\begin{array}{l}0 \\
\frac{8}{2} \\
\frac{1}{0} \\
0\end{array}$ & $\begin{array}{l}\frac{5}{5} \\
\frac{b}{i} \\
i\end{array}$ & $\begin{array}{l}\overline{8} \\
\frac{8}{8} \\
\frac{8}{5} \\
8 \\
8\end{array}$ \\
\hline 0.17 & & & & & & & & & & & & & & & & & & & & & & 0.17 \\
\hline 0.20 & & & & & & & & & & & $\begin{array}{c}0.79 \\
\ldots\end{array}$ & & & & & & & & & & & $\begin{array}{c}0.20 \\
\ldots\end{array}$ \\
\hline 4.47 & & & & & & & & & & & 0.79 & & & & & & & 0.15 & & & 0.60 & $\begin{array}{l}\ldots .47 \\
4.47\end{array}$ \\
\hline 5.12 & & & & & & & & & & & 0.70 & & & & & & & 0.37 & & & $1 . x$ & 5.12 \\
\hline 5.67 & & & & 0.17 & & & 0.28 & 0.21 & & & 0.70 & & & & & & & 0.04 & 0.30 & 0.47 & 3.00 & 5.67 \\
\hline 0.72 & & & & 0.60 & & & 0.74 & $0.5 \theta$ & & & 0.70 & & & & & & & 0.07 & 1.06 & 1.28 & 3.64 & 6.72 \\
\hline 7.70 & & & & 120 & & & 1.21 & 0.00 & & & 0.70 & & & & & & & 1.34 & 1,00 & 1.87 & 4.01 & 7.70 \\
\hline 6.82 & 0.11 & 0.10 & & 1.72 & 0.13 & & 1.54 & 1.25 & & & 0.79 & & & & & & & 1.70 & 2.44 & 2.30 & 4.24 & 8.62 \\
\hline 10.10 & 0.34 & 0.51 & & 2.11 & 0.41 & & 1.87 & 1.50 & 0.27 & & 0.70 & & & & & & & 2.20 & 2.80 & 2.00 & 4.49 & 10.10 \\
\hline 11.50 & 0.75 & 1.00 & & 2.01 & 0.94 & & 2.34 & 1.00 & 1.12 & & 0.70 & & & & & & & 2.06 & 3.45 & 3.14 & 4.92 & 11.56 \\
\hline 13.25 & 1.52 & t.76 & & 3.30 & 1.00 & & 3.11 & 2.47 & 2.08 & & 0.70 & & 0.54 & & & & & 3.10 & 4.22 & 9.42 & 8.73 & 13.25 \\
\hline 15.17 & 2.50 & 2.74 & 0.00 & 4.24 & 2.70 & 0.20 & 4.16 & 3.10 & 4.40 & 0.10 & 0.70 & 1.80 & 1.76 & 0.16 & 1.30 & $0.7 \pi$ & 1.30 & 3.54 & 1.05 & 4.65 & 7.30 & 15,17 \\
\hline 17.38 & 3.61 & 3.87 & 3.34 & 5.40 & 4.66 & 2.00 & 3.67 & 4.30 & 7.06 & 1.67 & 2.05 & 720 & 9.73 & 2.13 & 3.8t & 4.03 & 6.75 & 3.pes & 8.00 & 5.45 & 0.28 & 17.30 \\
\hline 12000 & s.18 & $4 . \infty$ & 3.60 & 0.78 & 0.43 & 5.25 & 7,13 & 5.03 & 0.75 & 4.20 & 1.00 & 9.00 & 4.50 & 4.36 & 4,30 & 0.17 & 9.29 & 4.43 & 6.08 & 0.11 & 19.28 & 19.90 \\
\hline 22.80 & 0.55 & 0.00 & 7.72 & 0.57 & t.1.5 & 7.56 & 0.73 & 7.59 & 10.43 & 8.97 & 8.20 & 10.03 & 8.00 & 600 & 4.40 & 7.42 & 10.35 & 8.02 & 6.64 & 0.00 & 13.56 & 22.00 \\
\hline 26.11 & 0.08 & 7.24 & 10.21 & $10.1 \mathrm{~s}$ & 10.21 & 10.04 & 10.30 & 9.10 & 19.10 & 8.10 & 9.06 & 1250 & 5.74 & 0.85 & 4.71 & 0.47 & 11.58 & s.7n & 7.73 & 0.16 & 16.35 & 28.11 \\
\hline 29.91 & 9.57 & 0.01 & 13.51 & 1227 & 11.00 & 10.14 & 1220 & 10.00 & 17.03 & 0.21 & 0.91 & 16.32 & 7.24 & 7.73 & 5.50 & 13.17 & 14.40 & 0.71 & 9.30 & 0.85 & 20.12 & 20.01 \\
\hline 34.25 & 11.20 & 10.00 & 17.20 & 14.17 & 13.21 & 20.15 & 14.15 & 12.04 & 24.78 & 10.27 & 10.53 & 23.40 & 10.02 & 0.45 & 0.28 & 19.61 & 19.82 & 7.85 & 11.7 & 1205 & 24.74 & 34.25 \\
\hline 39.23 & 13.11 & 19.75 & 19.77 & 16.27 & 14.70 & 22.02 & 10.34 & 13.37 & 31.15 & 11.18 & 11.10 & 33.22 & 13.54 & 0.23 & 15.85 & 25.63 & 28.90 & 0.21 & 13.90 & 14.07 & 20.44 & 39.23 \\
\hline 44.04 & 15.37 & 13.71 & 22.31 & 10.10 & 17,53 & 25.37 & 10.25 & 15.10 & 37.20 & 13.06 & 12.00 & 45.30 & 10.06 & 10.47 & 32.81 & 32.60 & 45.80 & 10.22 & 16.22 & 10.39 & 34.40 & M.M. \\
\hline 51,47 & 16.10 & 10,00 & 25.01 & 23,13 & 22.00 & 24.06 & 23.23 & 10.05 & 42.44 & 18.30 & 10.59 & 54.08 & 23.01 & 13.70 & 51.25 & 45.08 & 04.05 & 12.77 & 19.21 & 10.54 & 40.00 & 51.47 \\
\hline 58.05 & 21.66 & 10.00 & 27.54 & 27.10 & 20.40 & 31.10 & 27.30 & 23,05 & 47.70 & 20.30 & 20,60 & 81.14 & 27.60 & 16.60 & 63.72 & 51,03 & 77.15 & 15.10 & 22.50 & 23.14 & 45,70 & 50.05 \\
\hline 67.52 & 26.15 & 2280 & 34.34 & 3121 & 30.07 & 37.05 & 31.37 & 20.67 & 55.30 & 25.32 & 26.02 & 67.26 & 33.46 & 20.74 & 70.70 & 67.21 & 85.37 & 17.81 & 28.58 & 27.35 & 51.70 & 87.52 \\
\hline 77.34 & 31.78 & 27.11 & 30.73 & 35.22 & 34.01 & 40.01 & $\mathbf{3 6 . 0 0}$ & 33.15 & 69.26 & 30.13 & 30.00 & 74.55 & 41.64 & 24.40 & 75.03 & 87.81 & $\infty . \infty$ & 21.25 & 31.00 & 31.95 & s.5.57 & $\pi .34$ \\
\hline 80.50 & 38.48 & 32.80 & su.se & 42.50 & 41.52 & 75.54 & 43.10 & 37.51 & $\infty . \infty$ & 35.81 & 34.50 & 03.05 & 53,06 & 20.47 & B1.12 & 90.60 & 93.00 & 25.20 & 38.18 & 37.24 & 00.50 & st.58 \\
\hline 101.40 & 46.73 & 39.4 & the & 58.24 & 53.96 & 26.50 & 57.68 & 42.00 & 97.57 & 41.06 & 30.60 & 22.04 & 67.12 & 35.60 & 87.50 & 100.00 & 95.06 & 20.92 & 42.45 & 43.00 & 78.31 & 101.40 \\
\hline 118.21 & 56.83 & 40.92 & 9s.80 & 77.96 & 75,10 & 0.61 & 78.85 & 57.06 & 90.62 & 50.85 & 48.61 & 97.05 & 01.12 & 43.24 & 93.54 & & 90.60 & 35.26 & 59.44 & 55.42 & 05.23 & 110.21 \\
\hline 133.10 & 65.00 & 50.60 & 100.00 & 08.98 & 06.26 & 100.00 & 98.60 & 78.14 & 100.00 & 68.30 & 63.19 & 00.60 & 92.45 & 54.00 & 90.50 & & 07.60 & 41.10 & 60.61 & 71.63 & 03.06 & 139.10 \\
\hline 152.45 & 73.22 & 71.48 & 100.00 & 100.00 & 100.00 & & 100.00 & 88,14 & & 60.78 & 01.0 & 100.00 & 90.13 & 50.77 & 100,00 & & D0.01 & 40.75 & 03.45 & 0.74 & os.11 & 152.45 \\
\hline 174.62 & 80,80 & 83.80 & & & & & & 100.00 & & 06.27 & 00.74 & & 100.00 & 71.15 & & & 100.00 & 56.00 & 00.64 & $\infty \pi$ & 100.00 & 174.82 \\
\hline 200.00 & 87.20 & 93.25 & & & & & & & & 100.00 & 100.00 & & & 20.50 & & & & 70.37 & 100.00 & 100.00 & & 200.00 \\
\hline 229.00 & 92.37 & 94.33 & & & & & & & & & & & & 100.00 & & & & $\Delta 2.92$ & & & & 200.00 \\
\hline 262.30 & 95.89 & $\$ 0.70$ & & & & & & & & & & & & & & & & 92.83 & & & & 202.39 \\
\hline 300.52 & 97.68 & 90.60 & & & & & & & & & & & & & & & & 97.64 & & & & $\begin{array}{l}300.52 \\
344.21\end{array}$ \\
\hline $\begin{array}{l}344.21 \\
304.24\end{array}$ & $\begin{array}{l}08.56 \\
0.58\end{array}$ & 100,00 & & & & & & & & & & & & & & & & $\begin{array}{l}9.21 \\
9.63\end{array}$ & & & & $\begin{array}{r}34.21 \\
304.24\end{array}$ \\
\hline 451.50 & 9.83 & & & & & & & & & & & & & & & & & 99.87 & & & & 451.50 \\
\hline $\begin{array}{l}517.20 \\
502,39\end{array}$ & 100.00 & & & & & & & & & & & & & & & & & 100.00 & & & & $\begin{array}{l}517: 20 \\
502.30\end{array}$ \\
\hline
\end{tabular}

Table 4-6 lists the particle diameters corresponding to the $5^{\text {th }}, 25^{\text {th }}, 50^{\text {th }}, 75^{\text {th }}$, and $95^{\text {th }}$ percentile points of the cumulative PSDs given in Table 4-5. These particle diameters are known as the "quantiles" for the various percentile points of the cumulative distribution function. The quantiles corresponding to the $50^{\text {th }}$ percentile points are the medians of the distributions. The quantiles and percentiles were obtained from a linear interpolation between the numbers given in Table 4-5. This table also lists the mean of the quantiles for each of the three tanks.

Table 4-7 gives the tolerance limits and confidence limits on the mean percentiles. The three tank quantile means given in Table 4-6 are included in Table 4-7. The row called the "mean" is the arithmetic mean of the three means and "S.D." is the standard deviation of the three means. The bottom two rows of Table 4-7 are the 95/95TL and the UL(95\%). For example, based on the mean percentiles, $107 \mu \mathrm{m}$ is the estimate of the median particle diameter, $256 \mu \mathrm{m}$ is the estimate of $95 / 95 T L$ on the median, and $140 \mu \mathrm{m}$ is the estimate of the UL(95\%) on the median. 


\section{RPP-6427 REV 0}

Table 4-6. Estimates of Quantiles, Based on Linear Interpolation, for Five Percentile Points (units for quantiles are $\mu \mathrm{m}$ ).

\begin{tabular}{|c|c|c|c|c|c|}
\hline \multirow[b]{2}{*}{ Tank.Sample } & \multicolumn{5}{|c|}{ Percentlle Polints } \\
\hline & 5th & 25th & $50 t h$ & 75th & 95th \\
\hline AZ101.S99T001947 & 23 & 75 & 122 & 180 & 289 \\
\hline AZ101.S99T001948 & 20 & 73 & 118 & 162 & 212 \\
\hline Moan & 21 & 74 & 120 & 171 & 250 \\
\hline C104.T- & 26 & 100 & 178 & 242 & 322 \\
\hline C104.T0 & 16 & 64 & 111 & 141 & 168 \\
\hline C104.TPOS & 16 & 63 & 109 & 137 & 166 \\
\hline C104.WTS & 12 & 35 & 65 & 101 & 141 \\
\hline Mean & 18 & 66 & 116 & 155 & 199 \\
\hline AW103.S00T000386 & 19 & 51 & 83 & 95 & 110 \\
\hline AW103.S00T000387 & 16 & 56 & 95 & 115 & 130 \\
\hline AW103.S00T000388 & 18 & 57 & 97 & 116 & 131 \\
\hline AW103.S00T000389 & 20 & 44 & 77 & 89 & 100 \\
\hline AW103.500T000390 & 16 & 54 & 94 & 113 & 130 \\
\hline AW103.S00T000391 & 19 & 61 & 109 & 131 & 168 \\
\hline AW103.S00T000392 & 16 & 35 & 61 & 82 & 99 \\
\hline AW103.S99T001588 & 21 & 67 & 115 & 145 & 172 \\
\hline AW103.S99T001590 & 20 & 66 & 118 & 145 & 168 \\
\hline AW103.S99T001589 & 16 & 35 & 48 & 77 & 109 \\
\hline AW103.500T000508 & 23 & 54 & 85 & 110 & 143 \\
\hline AW103.S99T001592 & 21 & 77 & 126 & 180 & 217 \\
\hline AW103.S99T001593 & 28 & 42 & 51 & 76 & 123 \\
\hline AW103.S99T001594 & 19 & 39 & 58 & 71 & 85 \\
\hline AW103.S99T001587 & 17 & 37 & 46 & 57 & 100 \\
\hline Mean & 19 & 52 & 84 & 107 & 132 \\
\hline
\end{tabular}

Table 4-7. Tolerance Limits and Confidence Limits on Mean Percentiles (units are given in $\mu \mathrm{m}$ ).

\begin{tabular}{|l|c|c|c|c|c|}
\hline \multirow{2}{*}{ Tank } & \multicolumn{5}{|c|}{ Percentile Polnts } \\
\cline { 2 - 7 } & 5th & 25th & 50th & 75th & 95th \\
\hline AZ101.Mean & 21 & 74 & 120 & 171 & 250 \\
\hline C104.Mean & 18 & 66 & 116 & 155 & 199 \\
\hline AW103.Mean & 19 & 52 & 84 & 107 & 132 \\
\hline Mean & 19 & 64 & 107 & 144 & 194 \\
\hline S.D. & 2 & 11 & 19 & 33 & 59 \\
\hline S5/95TL & 34 & 151 & 256 & 400 & 647 \\
\hline UL(95\%) & 23 & 83 & 140 & 201 & 294 \\
\hline
\end{tabular}


The interpretations of the mean, the $95 / 95 \mathrm{TL}$, and the UL(95\%) are as follows:

- "Based on the analysis of mean percentiles, we estimate the median particle diameter in the HLW tanks to be $107 \mu \mathrm{m}$."

- "Based on the mean percentile estimate, we are $95 \%$ confident that the median particle size is less than $256 \mu \mathrm{m}$ for at least $95 \%$ of the population of HLW tanks."

- "Based on the mean percentile estimate, we are $95 \%$ confident that the average median particle size in the population of HLW tanks is less than $140 \mu \mathrm{m}$."

\subsubsection{Analysis of Variance (ANOVA)}

An alternative method to compute $95 \%$ confidence limits is to use the ANOVA. For each of the percentile points, a one-way ANOVA model was fit to the quantiles given in Table 4-6. The results from the ANOVA incorporate the variability between samples within tanks. This variability was not incorporated in the analysis of the means given above. The computer program S-PLUS $2000^{\mathrm{TM}}$ (S-PLUS 2000) was used to fit the ANOVA model to the data. For each percentile point, the ANOVA is used to estimate the mean quantile $(\mu \mathrm{m})$ and variance of the mean. These estimates are called Est.Mean and Var.Mean in Table 4-8. These two estimates were then used to construct UL(95\%), the upper limit to a one-sided $95 \%$ confidence interval on the median. For example, based on the ANOVA, $102 \mu \mathrm{m}$ is the estimate of the median particle diameter, and $139 \mu \mathrm{m}$ is the estimate of the UL(95\%) of the median. A tolerance limit cannot be computed.

Table 4-8. Confidence Limits on Percentiles Based on Analysis of Variance Estimates of Means and Variance of Means (Est.Mean and UL(95\%) are given in $\mu \mathrm{m}$; Var.Mean is given in $\mu \mathrm{m}^{2}$ ).

\begin{tabular}{|l|c|c|c|c|c|}
\hline Percentlle & 5 th & 25 th & 50th & 75 th & 95th \\
\hline Est.Mean & 19 & 61 & 102 & 139 & 189 \\
\hline Var.Mean & 1 & 46 & 161 & 420 & 1179 \\
\hline UL $(95 \%)$ & 21 & 81 & 139 & 199 & 289 \\
\hline
\end{tabular}

The interpretations of the mean and the UL(95\%) are as follows:

- "Based on the ANOVA, we estimate the median particle diameter in the HLW tanks to be $102 \mu \mathrm{m} . "$

- "Based on the ANOVA, we are 95\% confident that the average median particle size in the population of HLW tanks is less than $139 \mu \mathrm{m} . "$

Note that the data in the rows containing the mean values and UL(95\%) values in Table 4-8 are not very different from the data in the corresponding rows based on analysis of mean percentiles for the three tank means given in Table 4-7. This result indicates that there is little variability between the samples within the tanks. 


\section{RPP-6427 REV 0}

\subsubsection{Analysis of Tank Cumulative Means}

The third method for calculating mean quantiles and tolerance limits and confidence limits for those quantiles is based on the cumulative volume percent data from the PSD measurements. Table 4-4 listed the mean percent of the particle distribution in each size bin for each tank and for the mean of the three tank means. Table 4-9 gives the corresponding means, but on the cumulative volume percent scale (i.e., the percent of particulate volume that is less than the stated size). Table 4-10 gives estimates of the quantiles corresponding to the $5^{\text {th }}, 25^{\text {th }}, 50^{\text {th }}, 75^{\text {th }}$, and $95^{\text {th }}$ percentile points. These points were obtained by linear interpolation of values in Table 4-9.

Tolerance limits and confidence limits were computed using the three tank cumulative means. The limits are reported in the last two rows of Table 4-10. The data in the rows containing the means and the limits are not very different from the data in the corresponding rows in Table 4-7 and Table 4-8. For example, based on the tank cumulative means, $102 \mu \mathrm{m}$ is the estimate of the median particle diameter, $274 \mu \mathrm{m}$ is the estimate of 95/95TL on the median, and $140 \mu \mathrm{m}$ is the estimated of the UL( $95 \%)$ on the median.

The interpretations of the mean, the $95 / 95 T L$, and the UL(95\%) are as follows:

- "Based on the estimates of tank cumulative means, we estimate the median particle diameter in the HLW tanks to be $102 \mu \mathrm{m}$."

- "Based on the tank cumulative means estimate, we are $95 \%$ confident that the median particle size is less than $274 \mu \mathrm{m}$ for at least $95 \%$ of the population of HLW tanks."

- "Based on the tank cumulative means estimate, we are $95 \%$ confident that the average median particle size in the population of HLW tanks is less than $140 \mu \mathrm{m} . "$

\subsubsection{Summary of Statistical Analysis of Particle Size Data}

Table 4-11 gives a summary of the statistical results based on the three methods for analyzing the particle distribution data. The three methods were based on (1) an analysis of the mean percentile points, (2) an ANOVA, and (3) an analysis of the tank cumulative means. Tolerance limits cannot be computed based on the ANOVA method. Table 4-11 lists, by percentile point, the estimates of the mean particle diameter (i.e., three estimates plus an estimate based on the grand mean), estimates of the 95/95TL, and estimates of the UL(95\%). For each percentile point, the four methods for estimating the mean $(\mu \mathrm{m})$ give similar values, the two methods for estimating 95/95TL give similar values, and the three methods for estimating UL(95\%) give similar values. 


\section{RPP-6427 REV 0}

Table 4-9. Cumulative Particle Volume Distribution

Based on Tank Means (units are given in percentage).

\begin{tabular}{|c|c|c|c|c|}
\hline 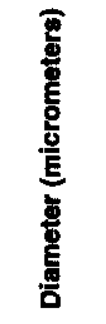 & 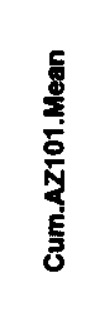 & 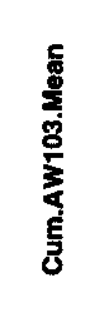 & 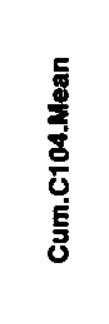 & 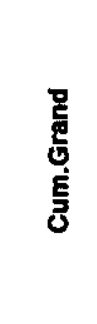 \\
\hline 0.20 & & 0.05 & & 0.02 \\
\hline & & $\cdots$ & & $\cdots$ \\
\hline 4.47 & & 0.05 & 0.15 & 0.07 \\
\hline 5.12 & & 0.05 & 0.53 & 0.19 \\
\hline 5.87 & & 0.10 & 1.03 & 0.38 \\
\hline 6.72 & & 0.19 & 1.65 & 0.61 \\
\hline 7.70 & 0.00 & 0.28 & 2.22 & 0.83 \\
\hline 8.82 & 0.09 & 0.36 & 2.60 & 1.02 \\
\hline 10.10 & 0.31 & 0.46 & 2.96 & 1.24 \\
\hline 11.56 & 0.68 & 0.64 & 3.43 & 1.58 \\
\hline 13.25 & 1.25 & 1.00 & 4.10 & 2.12 \\
\hline 15.17 & 2.13 & 1.84 & 5.02 & 3.00 \\
\hline 17.38 & 3.22 & 4.31 & 5.97 & 4.50 \\
\hline 19.90 & 4.40 & 6.24 & 6.86 & 5.83 \\
\hline 22.80 & 5.62 & 7.93 & 7.89 & 7.15 \\
\hline 26.11 & 6.90 & 9.47 & 9.31 & 8.56 \\
\hline 29.91 & 8.32 & 11.83 & 11.27 & 10.47 \\
\hline 34.25 & 9.83 & 15.08 & 13.82 & 12.91 \\
\hline 39.23 & 11.47 & 18.90 & 16.33 & 15.57 \\
\hline 44.94 & 13.41 & 24.51 & 19.08 & 19.00 \\
\hline 51.47 & 15.73 & 31.02 & 22.41 & 23.05 \\
\hline 58.95 & 18.57 & 36.68 & 26.04 & 27.10 \\
\hline 67.52 & 22.13 & 42.87 & 30.18 & 31.73 \\
\hline 77.34 & 26.63 & 50.03 & 34.88 & 37.18 \\
\hline 88.58 & 32.29 & 59.64 & 40.30 & 44.08 \\
\hline 101.46 & 39.31 & 70.30 & 46.71 & 52.11 \\
\hline 116.21 & 47.82 & 80.02 & 56.00 & 61.28 \\
\hline 133.10 & 57.76 & 89.53 & 67.24 & 71.51 \\
\hline 152.45 & 68.24 & 93.79 & 77.37 & 79.80 \\
\hline 174.62 & 78.56 & 97.81 & 87.04 & 87.80 \\
\hline 200.00 & 87.03 & 99.11 & 89.52 & 91.88 \\
\hline 229.08 & 92.81 & 100.00 & 92.59 & 95.13 \\
\hline 262.38 & 96.03 & & 95.73 & 97.25 \\
\hline 300.52 & 97.86 & & 98.23 & 98.70 \\
\hline 344.21 & 98.94 & & 99.41 & 99.45 \\
\hline 394.24 & 99.48 & & 99.80 & 99.76 \\
\hline 451.56 & 99.76 & & 99.91 & 99.89 \\
\hline 517.20 & 99.91 & & 99.97 & 99.96 \\
\hline 592.39 & 100.00 & & 100.00 & 100.00 \\
\hline
\end{tabular}


Table 4-10. Confidence Limits on Percentiles

Based on Tank Cumulative Means

(units are given in $\mu \mathrm{m}$ ).

\begin{tabular}{|l|c|c|c|c|c|}
\hline & \multicolumn{5}{|c|}{ Percentile Points } \\
\cline { 2 - 6 } & 5th & 25 th & 50th & 75th & 95th \\
\hline Cum.Grand.Mean & 18 & 55 & 99 & 141 & 229 \\
\hline Tank Mean & & & & & \\
\hline Cum.AZ101.Mean & 21 & 74 & 121 & 168 & 252 \\
\hline Cum.AW103.Mean & 18 & 46 & 77 & 109 & 161 \\
\hline Cum.C104.Mean & 15 & 57 & 107 & 149 & 256 \\
\hline Mean & 18 & 59 & 102 & 142 & 223 \\
\hline S.D. & 3 & 15 & 22 & 30 & 54 \\
\hline 95/95TL & 42 & 170 & 274 & 373 & 633 \\
\hline UL(95\%) & 24 & 83 & 140 & 193 & 313 \\
\hline
\end{tabular}

Table 4-11. Comparison of Percentile Means, Tolerance Limits, and Confidence Limits (units are given in $\mu \mathrm{m}$ ).

\begin{tabular}{|l|c|c|c|c|c|}
\hline & \multicolumn{5}{|c|}{ Percentile Points } \\
\cline { 2 - 7 } & 5th & 25th & 50th & 75th & 95th \\
\hline & \multicolumn{5}{|c|}{ Means } \\
\hline Mean Percentiles & 19 & 64 & 107 & 144 & 194 \\
\hline Analysls of Variance & 19 & 61 & 102 & 139 & 189 \\
\hline Tank Cumulatlve Means & 18 & 59 & 102 & 142 & 223 \\
\hline Cum.Grand.Mean & 18 & 55 & 99 & 141 & 229 \\
\hline & Tolerance Limits 95/95TL & 400 & 647 \\
\hline Mean Percentiles & 34 & 151 & 256 & NA & NA \\
\hline Analysls of Variance & NA & NA & NA & 633 \\
\hline Tank Cumulatlve Means & 42 & 170 & 274 & 373 & \\
\hline & Confidence Limits UL(95\%) & & 294 \\
\hline Mean Percentiles & 23 & 83 & 140 & 201 & 289 \\
\hline Analysis of Varlance & 21 & 81 & 139 & 199 & 313 \\
\hline Tank Cumulative Means & 24 & 83 & 140 & 193 & \\
\hline
\end{tabular}

In summary, based on PSD data from three HLW tanks:

- The estimate of the average median particle size diameter is approximately $110 \mu \mathrm{m}$.

- The TL95/95 on the median is approximately $275 \mu \mathrm{m}$.

- The UL(95\%) on the average median diameter is approximately $140 \mu \mathrm{m}$.

Table 4-11 gives similar estimates of particle size diameters corresponding to other percentiles (the $5^{\text {th }}, 25^{\text {th }}, 75^{\text {th }}$ and $95^{\text {th }}$ percentiles). In addition, a "typical" PSD (i.e., the distribution based on all the means) is included in the Grand.Mean column in Table 4-4. 


\section{RPP-6427 REV 0}

The UL(95\%) are estimates of the upper limit for the average median particle size diameter in the population of the HLW tanks. That is, if all the HLW were combined, the median particle diameter in the population would be less than $140 \mu \mathrm{m}$, with $95 \%$ confidence. However, because the waste will be processed one tank at a time, statistical results are needed that refer to the PSD in each tank. These are the 95/95TL values. The values calculated for the tolerance limit on the average median indicate that we are $95 \%$ confident that the median particle size diameter will not exceed $275 \mu \mathrm{m}$ in $95 \%$ of the HLW tanks. Thus, assuming that the PSD data are from a random sample of the HLW tanks, it is reasonable to use the bounding value for the median particle size of $275 \mu \mathrm{m}$ as the design basis for the WFD system. 


\section{RPP-6427 REV 0}

This page intentionally left blank. 


\section{RPP-6427 REV 0}

\subsection{CONCLUSIONS}

In this document, particle size measurements of the waste in Hanford Site tanks have been collected and assessed. A statistical analysis of these measurements was performed to provide a bounding value that may be used to refine the analysis of required flow rates and pressures in the WFD transfer system. The important findings of this process are summarized in this section.

\subsection{PARTICLE SIZE ASSESSMENT}

The Horiba ${ }^{\mathrm{TM}}$ Model LA-910 yielded the largest particle size values of all the instruments used to measure the size of particles in the HLW. The larger particle size results likely are results of agglomeration resulting from the high ionic strength of the suspension liquid invariably used with the Horiba ${ }^{T M}$ Model LA-910. The higher the ionic strength of the liquid in which particles are suspended, the higher the likelihood that agglomeration will occur. The results also may be related to the improved stirring capability of the Horiba ${ }^{\mathrm{TM}}$ Model LA-910 in relation to the other particle size measurement instruments. The improved stirring capability may bring large, heavy particles into the sensing region of the instrument more effectively than the stirring mechanisms in the other instruments may.

Many of the measurements made over the years probably did not register these large particles because the agglomerates were dispersed as a result of the low ionic strength of the suspension liquid (water or water/glycerin mixture) used in the measurements. Also, the upper particle size limit for at least one of the other instruments (the Microtrac ${ }^{\mathrm{TM}}$ Model UPA) was too small to register large agglomerates.

Whether the solids will remain agglomerated during transfer is an important question. The few laboratory studies that have been done indicate that agglomerates are broken up only slowly, if at all, by mechanical action. The agglomerates probably re-form rapidly after they are broken. Effective control of agglomeration probably can be attained only by adjustment of the ionic strength. Although laboratory tests cannot be compared directly with the effects expected from pumps, it is not prudent, with data now available, to assume that the agglomerates detected in PSD measurements will be broken up by pumps before transfer.

\subsection{CALCULATION OF DESIGN BASIS VALUE}

Because the results of particle size measurements taken using the Horiba ${ }^{\mathrm{TM}}$ Model LA-910 were higher than those taken with other instruments and because no reason was found to reject them, the Horiba ${ }^{\text {TM }}$ Model LA-910 results were selected as the basis for calculating a conservative upper limit for the particle sizes in the wastes. This upper limit is suitable as a design basis for the WFD transfer system. Because HLW contains the only solids that will be transferred over long distances, the design basis calculations were based only on the data obtained for HLW. These calculations lead to the conclusion that, with $95 \%$ confidence, the median particle size is not expected to exceed $275 \mu \mathrm{m}$ in at least $95 \%$ of the HLW tanks. 
RPP-6427 REV 0

This page intentionally left blank. 
RPP-6427 REV 0

\subsection{RECOMMENDATIONS}

Although this document provides a bounding median particle size for use in the design basis for the WFD transfer system, uncertainties remain regarding the size of the particles in the waste stored in underground tanks at the Hanford Site. The remaining issues are as follows:

- Uncertainty about particle sizes in the wastes because of the distinct, but unresolved, difference between results obtained with the Horiba ${ }^{\mathrm{TM}}$ Model LA-910 and results obtained with other instruments

- Uncertainty about the extent to which agglomerates in HLW slurries will be present during transport.

In addition to particle size, particle density is another characteristic affecting the transport properties of slurry. The initial objectives of this work did not address particle density, but discussions about agglomeration led to the recognition that the particle densities used in the WFD system analysis (RPP-5346) may be greater than they need be. Reduction of the particle density value used in the slurry flow modeling will result in lower required velocities and pipeline pressures.

If the uncertainties about particle size, agglomeration, and particle density can be reduced, it may be possible to reduce costs for design, construction, and qualification of the WFD transfer system. A change to the program baseline has been requested to resolve these uncertainties. The proposed work includes further literature assessments, additional laboratory work, and additional modeling of the transfer system.

\subsection{LITERATURE ASSESSMENTS}

The proposed literature assessments would be conducted along three lines of inquiry. First, literature related to industrial processes involving insoluble metal oxides and hydroxides would be reviewed to determine the particle sizes typically encountered when these materials are precipitated from aqueous solutions. Second, a review of Hanford Site literature would be conducted to verify the argument made in this document that the wastes in the three HLW tanks for which PSD has been measured with the Horiba ${ }^{\text {TM }}$ Model LA-910 can be used to represent all the HLW tank waste. And third, an attempt would be made to correlate the densities of particles settling in a tank or being transported in a pipe to densities that have been measured on settled sludge. 


\subsection{LABORATORY WORK}

The proposed laboratory work is of two types. Some of the studies are directed toward better understanding of the characteristics of the Horiba ${ }^{\mathrm{TM}}$ Model LA-910 and validating the associated measurement method. The other studies are aimed at elucidating characteristics of the particulate matter itself.

\subsubsection{Validation of the Measurement Process}

This group of tasks is proposed to further demonstrate that the Horiba ${ }^{\mathrm{TM}}$ Model LA-910 measurement method accurately reflects size distributions for several different kinds of particles. If authorized, the tests would include measurements with additional particle size standards. In addition particle-size measurements would be taken on actual and identical waste samples using the Horiba ${ }^{\mathrm{TM}}$ Model LA-910 and other instruments, and the results would be compared.

The Horiba ${ }^{\mathrm{TM}}$ Model LA-910 normally has been calibrated only with monodisperse standards (i.e., standards containing a very tight distribution of particle sizes). Polydisperse standards (i.e., standards with well-characterized, wide particle distributions) can be obtained commercially. It is proposed that these standards be used to demonstrate that the proprietary mathematical conversion of the light-scattering data to PSD is performed correctly on polydisperse samples as well as monodisperse ones. For comparison, an instrument at Pacific Northwest National Laboratory that has historically produced results considerably lower than the results produced using the Horiba ${ }^{\mathrm{TM}}$ Model LA-910 should be tested using the same standards.

Samples of HLW should be obtained from the sample archives at the 222-S Laboratory for headto-head comparisons of measurements taken using the Horiba ${ }^{\mathrm{TM}}$ Model LA-910 with measurements taken using the instrument at Pacific Northwest National Laboratory. Special care should be taken to provide identical samples for each instrument. Previous comparisons, mentioned earlier in this document, have not had the benefit of using samples with the same history of treatment and storage. Care should be taken to obtain the PSD measurements on the two instruments under the same conditions of ionic strength, $\mathrm{pH}$, and other chemical parameters. Insofar as possible, identical instrument parameters should be used for the measurements on the two instruments. The ionic strength, rate and duration of stirring, and application of ultrasonic energy to the samples before measurement should be varied for both instruments according to a predetermined plan to evaluate the sensitivity of the measurements to these variables.

\subsubsection{Characterization of Waste Particles}

Another group of tests is proposed to expand the understanding of the nature of the particulate matter in the wastes. Characteristics such as agglomeration, particle shape, particle density, and other factors that affect settling rate would be studied in addition to settling rate and particle size per se. These studies would assist in understanding the limitations of particle size measurements and modeling efforts alike. 
Some simple sieving tests are proposed, using sieves in the range of $50 \mu \mathrm{m}$ to $200 \mu \mathrm{m}$. These tests would determine whether hard, granular particles greater than the mesh size of the sieve exist in the sample. However, if the particles are soft agglomerates, they may be sheared by the sieve and simply re-agglomerate after sieving. Polarized light microscopy and PSD testing of the sieved and unsieved particles, possibly at various times after sieving and before and after sonication, can determine the extent to which sieving changes the character of the particles and their size distribution. Such data can be used to understand the susceptibility of agglomerates to mechanical disruption and re-agglomeration.

Several types of settling tests have been proposed. "Leading-edge" settling tests, in which the settling rate of the fastest-settling particles is measured, could be performed. Although these tests would not provide information about the majority of the particles in the waste, they would provide information about the particles that are the most difficult to transfer. Combined with microscopy and Stokes Law, these tests would provide an estimate of the density of typical agglomerated particles in the waste. Further information about the densities of the particles could be obtained by fractional settling tests. In these tests, the waste would be classified into three or more fractions by settling, and each of the fractions will be subjected to polarized light microscopy and PSD.

Polarized light microscopy would be a valuable tool in the proposed studies. In addition to the information regarding particle sizes and extent of agglomeration, polarized light microscopy would yield information about the types of particles present and a general idea about the size distribution of each type. These kinds of observations would help to understand the limitations of PSD measurements.

The results of these tests would provide a basis for reviewing and validating the PSD measurement method. Alternatively, the results would be used to revise the analytical method and/or the design basis value determined in this document.

\subsection{MODELING OF THE TRANSFER SYSTEM}

Recalculation of the required slurry flows and pipe pressures presented in RPP-5346 is recommended to incorporate the findings of the literature and laboratory studies. A Monte Carlo calculation is recommended to estimate the uncertainties in the results of these calculations. These analyses will determine the minimum allowable design pressures for slurry transfers. 
RPP-6427 REV 0

This page intentionally left blank. 


\section{RPP-6427 REV 0}

\subsection{REFERENCES}

Allen, T., 1997, Particle Size Measurement, Volume 1, "Powder Sampling and Particle Size Measurement," $5^{\text {th }}$ ed., Chapman \& Hall, London, England.

BNFL-RPT-030, 2000, Characterization, Washing, Leaching, and Filtration of C-104 Sludge, Rev. 0, BNFL Inc., Richland, Washington.

Bott, S. E., and W. H. Hart, 1991, “Extremely Wide Dynamic Range, High-Resolution Particle Sizing by Light Scattering," Chapter 7 in Particle Size Distribution II. Assessment and Characterization, ACS Symposium Series 472, American Chemical Society, Washington, D.C.

DOE, 2000, Tank Characterization Database, data part of the Tank Waste Information Network System, on the internet at http://twins.pnl.gov:8001/, (U.S. Department of Energy, Washington, D.C.

Gray, W. J., M. E. Peterson, R. D. Scheele, and J. M. Tingey, 1993a, Characterization of the First Core Sample of Neutralized Current Acid Waste from Double-Shell Tank 102-AZ, unnumbered report, Pacific Northwest Laboratories, Richland, Washington.

Gray, W. J., M. E. Peterson, R. D. Scheele, and J. M. Tingey, 1993b, Characterization of the Second Core Sample of Neutralized Current Acid Waste from Double-Shell Tank 101-AZ, unnumbered report, Pacific Northwest Laboratories, Richland, Washington.

HNF-1647, 1999, Tank 241-S-111, Core 237 Analytical Results for the Final Report, Rev. 0A, Waste Management of Hanford, Inc., for Fluor Daniel Hanford, Inc., Richland, Washington.

HNF-1650, 1998, Tank 241-U-109, Core 238 Analytical Results for the Final Report, Rev. 0, Waste Management of Hanford, Inc., for Fluor Daniel Hanford, Inc., Richland, Washington.

HNF-1666, 1999, Tank 241-SY-101, Cores 255, 256, and 257, Analytical Results for the Final Report, Rev. 0A, Waste Management of Hanford, Inc., for Fluor Daniel Hanford, Inc., Richland, Washington.

HNF-2177, 1998, Tank-by-Tank Safety Status Evaluation, Rev. 0-B, Lockheed Martin Hanford Corporation, Richland, Washington.

HNF-3352, 1998, Results of Dilution Studies with Waste from Tank 241-AN-104, Rev. 0, Numatec Hanford Corporation for Lockheed Martin Hanford Corporation, Richland, Washington.

HNF-4964, 2000, Results of Dilutions Studies with Waste from Tank 241-AW-101, HNF-4964, Rev. 0A, Numatec Hanford Corporation for CH2M HILL Hanford Group, Inc., Richland, Washington. 


\section{RPP-6427 REV 0}

HNF-SD-WM-DTR-046, 1999, Results of Dilution Studies with Waste from Tank 241-AN-105, Rev. OA, Numatec Hanford Corporation for Lockheed Martin Hanford Corporation, Richland, Washington.

HNF-SD-WM-SP-012, 2000, Tank Farm Contractor Operation and Utilization Plan, Rev. 2, CH2M HILL Hanford Group, Inc., Richland, Washington.

Jewett, J. R., 2000, Work Plan to Reduce Uncertainty of Particle Size Estimates, (Interoffice Memo 6N100-00-048 to P. J. Certa, dated April 21), Numatec Hanford Corporation, Richland, Washington.

LA-UR-97-2889, 1997, Sludge Washing and Alkaline Leaching Tests on Actual Hanford Tank Sludge: FY 1997 Results, Los Alamos National Laboratory, Los Alamos, New Mexico.

National Bureau of Standards Handbook 91, 1963, "Experimental Statistics," U.S. Department of Commerce, Washington, D.C.

O'Rourke, J. F., 1996, C-106 Viscosity and Particle Size Distribution Results, (Internal Memo 75764-PCS96-050 to R. A. Esch, Westinghouse Hanford Company, dated May 22) in Process Aids, Vol. 28, HNF-IP-0711-28, Rev. 0, Numatec Hanford Corporation, Richland, Washington.

O'Rourke, 2000a, Particle Size Distribution Analyses for Tank 241-AZ-101, (Interoffice Correspondence 8D500-JFO-00-017 to F. H. Steen, dated April 13), Fluor Hanford, Inc., Richland, Washington.

O'Rourke, 2000b, Physical Property Measurements for Tanks 241-AW-103, 241-AZ-101, and 241-AZ-102, (Interoffice Correspondence 8D500-JFO-00-011 to F. H. Steen, dated March 2), Fluor Hanford, Inc., Richland, Washington.

Peters, B., 1988, Tank 101-AY Sludge Particle Size Analysis, (unnumbered memorandum to R. S. Edrington, dated July 22), Westinghouse Hanford Company, Richland, Washington.

Peterson, M. E., 1989, Final Report--Characterization of the First Core Sample of Neutralized Current Acid Waste from Double-Shell Tank 101-AZ, (unnumbered letter to A. J. DiLiberto, dated September 29), Pacific Northwest Laboratories, Richland, Washington.

Peterson, M. E., 1990, Preliminary Results-Analysis of Waste Layers from DST 101-AZ Core \#2, (Letter 9001040 to A. J. DiLiberto and L. M. Sasaki, dated February 28), Pacific Northwest Laboratories, Richland, Washington.

PNL-10712, 1995, Washing and Caustic Leaching of Hanford Tank Sludges: Results of FY 1995 Studies, Pacific Northwest Laboratories, Richland, Washington.

PNL-10761, 1995, Effect of Colloidal Aggregation on the Sedimentation and Rheological Properties of Tank Waste, Pacific Northwest Laboratories, Richland, Washington. 


\section{RPP-6427 REV 0}

PNNL-11278, 1996, Washing and Caustic Leaching of Hanford Tank Sludges: Results of FY 1996 Studies, Rev. 1, Pacific Northwest National Laboratory, Richland, Washington.

PNNL-1 1352, 1996, Tank SY-102 Waste Retrieval Assessment: Rheological Measurements and Pump Jet Mixing Simulations, Pacific Northwest National Laboratory, Richland, Washington.

PNNL-11381, 1996, Washing and Caustic Leaching of Hanford Tank C-106 Sludge, Pacific Northwest National Laboratory, Richland, Washington.

PNNL-11636, 1997, Washing and Caustic Leaching of Hanford Tank Sludge: Results of FY 1997 Studies, Pacific Northwest National Laboratory, Richland, Washington.

RPP-5346, 2000, Waste Feed Delivery Transfer System Analysis, Rev. 0, CH2M HILL Hanford Group, Inc., Richland, Washington.

RPP-5798, 2000, Results of Retrieval Studies with Waste from Tank 241-C-104, Rev. 0, CH2M HILL Hanford Group, Inc., Richland, Washington.

RPP-6548, 2000, Preliminary Test Report, 241-AZ-101 Mixer Pump Test, Rev. 0, CH2M HILL Hanford Company, Inc., Richland, Washington.

Sennett, P., and J. P. Olivier, 1965, "Colloidal Dispersions, Electrokinetic Effects, and the Concept of Zeta Potential," Chemistry and Physics of Interfaces, pp. 73-92, American Chemical Society, Washington, D.C.

S-PLUS, 2000, S-PLUS 2000, Data Analysis Products Division, MathSoft, Inc., Seattle, Washington.

WHC-SD-WM-TI-540, 1994, Analysis Report for 241-BY-104 Auger Samples, Rev. 1, Westinghouse Hanford Company, Richland, Washington. 
RPP-6427 REV 0

This page intentionally left blank. 


\section{RPP-6427 REV 0}

\section{APPENDIX A}

INDEPENDENT REVIEW OF CALULATIONS 


\section{RPP-6427 REV 0}

This page intentionally left blank. 
RPP-6427 REV 0

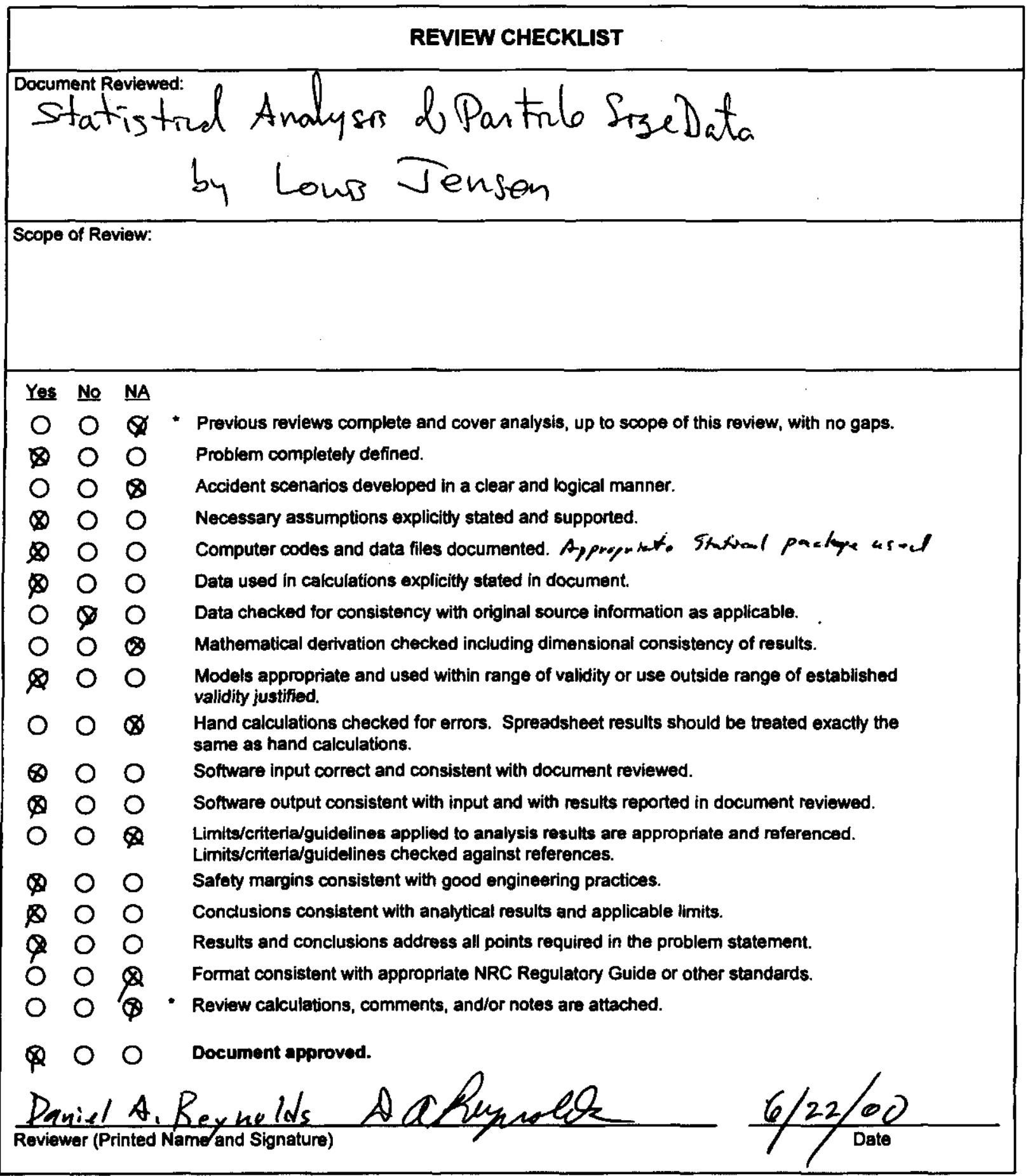

"Any calculations, comments, or notes generated as part of this review should be signed, dated and attached to this checklist. Such material should be labeled and recorded in such a manner as to be intelligible to a technically qualified third party. 


\section{RPP-6427 REV 0}

This page intentionally left blank.

A-2 


\section{DISTRIBUTION}

\section{Onsite}

11

CH2M HILL Hanford Group, Inc.

Barton, W. B., III

R2-11

Bechtold, R. A.

K7-22

Field, J. G.

R2-12

Hunt, J. W.

R2-12

Jensen, L. (3)

R2-12

Julyk, L. J.

R3-83

Kirch, N. W.

R2-11

Oten, T. C.

R3-83

Parker, D. L.

R3-75

5

Lockheed Martin Services, Inc.

Rice, R. L. (3)

R3-73

Central Files

Document Processing Center

Numatec Hanford Corporation

Carlson, A. B.

R3-73

Certa, P. J.

R3-73

Choho, A. F.

R3-73

Conrads, T. J.

Galbraith, J. D.

R3-73

Garfield, J. S.

R3-73

R3-73

Grenard, C. E.

R3-73

Jewett, J. R. (5)

R3-73

Senentz, G.

R3-73

Willis, W. L.

R3-73

\section{Subcontractors}

Bechtold, D. B., ANL SVC

T6-07

Callaway, W. S., III, COGEMA

Herting, D. L., ANL SVC

T6-07

Lockrem, L. L., ANL SVC

T6-07

\section{U.S. Department of Energy}

Richland Operations Office

Reading Room

H2-53

Distr.-1 
RPP-6427 REV 0

DISTRIBUTION (continued)

$\underline{\text { Offsite }}$

1

Oak Ridge National Laboratory

C. P. McGinnis

Oak Ridge National Laboratory

P.O. Box 2008, MS6178

Oak Ridge, Tennessee 37831-6178

1

Washington State University

K. C. Liddell

Department of Chemical Engineering

Washington State University

Pullman, Washington 99164-2710

Distr.-2 